ESAIM: COCV 19 (2013) 906-929

DOI: $10.1051 / \mathrm{cocv} / 2012038$
ESAIM: Control, Optimisation and Calculus of Variations

www.esaim-cocv.org

\title{
HOMOGENEOUS APPROXIMATIONS AND LOCAL OBSERVER DESIGN
}

\author{
Tomas Ménard ${ }^{1}$, Emmanuel MoulaY $^{2}$ and Wilfrid Perruquetti ${ }^{3}$
}

\begin{abstract}
This paper is concerned with the construction of local observers for nonlinear systems without inputs, satisfying an observability rank condition. The aim of this study is, first, to define an homogeneous approximation that keeps the observability property unchanged at the origin. This approximation is further used in the synthesis of a local observer which is proven to be locally convergent for Lyapunov-stable systems. We compare the performance of the homogeneous approximation observer with the classical linear approximation observer on an example.
\end{abstract}

Mathematics Subject Classification. 93B07, 93B29, 16 W25.

Received April 10, 2012. Revised September 25, 2012.

Published online June 3, 2013.

\section{INTRODUCTION}

Observers design for nonlinear systems has been an active research area for the last decades. Explicit constructions for nonlinear systems have been investigated, for example high gain observers (see $[8,9,16,20])$ or backstepping observers (see $[2,19])$. But these constructions only apply to very specific class of systems. That is why many tools have been developed in order to transform systems into a suitable form for observer design.

The first results have been obtained using differential geometry. Necessary and sufficient conditions for exact linearization of nonlinear systems can be found in [17] for systems without inputs and in [18] for systems with inputs. These papers give explicit change of coordinates, nevertheless there is two restrictions to their application. First, the class of systems which are exactly linearizable is restrictive. Second, even if the conditions are met, the computation of the transformation becomes quickly very heavy as the dimension increases, see [22].

A theory which has similar considerations has been developed in the early 90 's, see [6,7] for recent developpment. The algebraic observability characterizes systems for which the state can be written as a function of the input and output and a finite number of their derivatives. This theory uses differential algebra, and instead of solving differential equations to find the transformation, we need to solve algebraic equations. Just as exact linearization, these equations can be very complex for nonlinear systems.

Keywords and phrases. Homogeneity, approximations, local observer.

1 GREYC, UMR CNRS 6072, Université de Caen, 6 Bd du Maréchal Juin, BP 5186-14032 Caen Cedex, France.

tomas.menard@unicaen.fr

2 Xlim-SIC, UMR CNRS 6172, Université de Poitiers, Bvd Marie et Pierre Curie, BP 30179, 86962 futuroscope Chasseneuil, France. emmanuel.moulay@univ-poitiers.fr

3 NON-A, INRIA Lille Nord Europe and LAGIS UMR CNRS 8219, Ecole Centrale de Lille, BP 48, 59651 Villeneuve D'Ascq, France. wilfrid.perruquetti@ec-lille.fr 
The restrictions of the previous approaches have been alleviated since then. Conditions for the transformation of nonlinear systems into more general forms than exact linear systems have been explored. These classes of systems consists in systems which are made up of a particular linear part and a nonlinear part satisfying some conditions, and are characterized using algebro-geometric tools, see $[3,5,10,21,24]$ for example. The common point in all these approaches is that the output function is always linearized, hence, this still restrict the class of systems considered while it allows to obtain global or semi-global convergence.

Another construction which does not require to linearize the output function has been developed in [15], it uses the Lyapunov auxiliary Theorem and a direct coordinate transformation. Conditions for the existence of this transformation have been further study, especially in [1]. The main drawback is that it requires to solve a set of partial differential equations and is then not constructible in general.

An alternative method is to consider approximations and to design the observer considering only a part of the system. This allow to reduce the complexity of the system and then to actually design an observer. But, generally, only local convergence is obtained for the observer. Nevertheless, if the considered system is too complex, this can be the only possibility since other methods cannot be applied in practice.

Some of the previous approaches cited here, especially the one derived from exact linearization, can be viewed as approximation design, since the observer is designed using the linear part only while the conditions on the nonlinear part ensure convergence of the observer. Here, we are interested in a dedicated approach to approximation for the observability problem, which applied to a wide class of systems, that is systems verifying an observability rank condition. Unlikely to the works derived from the exact linearization, here we aim at designing an approximation for both the vector field and the output function.

The simplest strategy when the system is too complex is the linear approximation. It gives an efficient procedure to design a local observer and apply to a wide class of systems. Two options are available, we can consider the linear approximation in the neighborhood of a point or along a trajectory. Although the linear approximation along a trajectory leads to better results, a very few results exist about the convergence and lead to very restrictive conditions. Thus, we consider here approximation in the neighborhood of a point, without loss of generality, we assume that this point is the origin.

We believe that a more efficient approximation can be designed, since the linear one is not directly induced by the problem of observability. We want to construct an approximation which is specially adapted to the observability problem. In order to obtain such an approximation, we consider the tool of homogeneity which gives the necessary freedom for this construction. This tool has already been used for the construction of homogeneous approximations for controlled systems and has been worked out by numerous authors $[4,12,13,25,27]$. It is rooted from the theory of hypo-elliptic operators [11,23]. It has been proved that for any nonlinear control system which is fully accessible, there always exists an homogeneous approximation which remains fully accessible, see [13]. More precisely, given a control system

$$
\dot{x}=f_{0}(x)+\sum_{i=1}^{m} u_{i} f_{i}(x), \quad x \in \mathbb{R}^{n},
$$

the rank of the Lie algebra spanned by the vector fields $f_{0}, \ldots, f_{m}$ is assumed to be $n$. A change of coordinates is derived from a suitable filtration of this Lie algebra. The homogeneous approximation of system $(\Sigma)$ is then obtained by taking the first term in the homogeneous expansion of the vector fields $\left\{f_{0}, f_{1}, \ldots, f_{m}\right\}$ written in the new system of coordinates.

To the best of our knowledge, the dual problem of the existence of an observable homogeneous approximation for an observable nonlinear system has not been investigated yet. This problem is solved next (locally at the origin). It is shown that this homogeneous approximation always exists and is explicitly constructed. Similarly to the case of controllability we construct a flag on the space of observability, which is spanned by the oneforms associated to the output functions and their Lie derivative along the vector field of the system. There are two main issues which make the difference between accessible homogeneous approximation and observable homogeneous approximation. First, the space of observability is a subspace of the space of one-forms on $\mathbb{R}^{n}$ and 
not the algebra of vector fields. Second, an homogeneous approximation has to be constructed that copes with both state equation and output equation. The resulting system has to consist simultaneously of an approximation for the vector fields and of an approximation for the output function.

The approximation obtained here is further used in order to construct an observer for a general class of nonlinear systems. Sufficient conditions are given for the convergence of the obtained observer. The construction presented here is compared with the linear approximation observer. The performance of both methods is illustrated by an example.

The paper is organized as follows. In Section 2, definitions and notations are given, which will be used in the paper. Section 3 contains the main theoretic development of this paper. The approximation induced by the observability property of a system without inputs is constructed. Properties of the space of observation of the approximating system are stated. An observer is designed using the previously constructed approximation, in Section 4. Sufficient conditions for the convergence of this observer are stated. In Section 5, an example is given which illustrate the performance of the observers obtained by a linear approximation and an homogeneous approximation. Section 6 contains the proofs. Finally, the conclusion is given in Section 7.

\section{Definitions And notations}

\subsection{Notations}

In the sequel, we denote:

- $\mathbb{R}$ the set of real numbers;

- $\mathbb{N}$ the set of nonnegative integers;

- "span" the linear span on the corresponding vector space over the field $\mathbb{R}$;

- $\mathcal{L}_{f} h$ the Lie derivative of the function $h: \mathbb{R}^{n} \rightarrow \mathbb{R}$ along the vector field $f: \mathbb{R}^{n} \rightarrow \mathbb{R}^{n}$;

- $[f, g]$ the Lie bracket of the vector fields $f, g: \mathbb{R}^{n} \rightarrow \mathbb{R}^{n}$;

- $\operatorname{ad}_{f}^{i} g$, where $i \in \mathbb{N}$ is defined by induction with $\operatorname{ad}_{f}^{0} g=g$ and $\operatorname{ad}_{f}^{i}=\left[f, \operatorname{ad}_{f}^{i-1} g\right]$ for $i \geq 1$;

- $\omega(g)(x) \triangleq \omega(x)(g(x)), x \in \mathbb{R}^{n}$, where $\omega$ is a 1 -form on $\mathbb{R}^{n}$ and $g$ is a vector field on $\mathbb{R}^{n}$;

- $(t, \xi) \rightarrow \exp (t f)(\xi)$ the solution of $\dot{x}(t)=f(x(t)), x(0)=\xi \in \mathbb{R}^{n}$, where $f: \mathbb{R}^{n} \rightarrow \mathbb{R}^{n}$ is a smooth vector field;

- an open subset $\mathcal{U}$ of $\mathbb{R}^{n}$ is said to be positively invariant under system $\dot{x}(t)=f(x(t))$ if every solution $x(t)$ of this system starting in $\mathcal{U}$ stays in $\mathcal{U}$;

- $\|.\|_{i, k}$ the $i$-norm on $\mathbb{R}^{k}$, where $i, k \in \mathbb{N}$ (if not specified, norm 2 will be used);

- $\|\cdot\|_{M}$ the norm associated to the symmetric definite positive matrix $M$;

- $\mathcal{B}_{\|.\|}(x, \epsilon)=\left\{z \in \mathbb{R}^{n} \mid\|z-x\|<\epsilon\right\}$, where $x \in \mathbb{R}^{n}, \epsilon>0$ and $\|$.$\| is a norm on \mathbb{R}^{n}$;

- $\delta_{i}^{j}$ the usual Kronecker symbol which equal 1 if $i=j, 0$ otherwise;

- $|\alpha|=\sum_{i=1}^{n} \alpha_{i}$, where $\alpha \in \mathbb{N}^{n}$ is a multi-index;

- $\|\alpha\|_{r}=\sum_{i=1}^{n} r_{i} \alpha_{i}$, where $\alpha, r \in \mathbb{N}^{n}$

- $z^{\alpha}=z_{1}^{\alpha_{1}} \ldots z_{n}^{\alpha_{n}}$, where $\alpha \in \mathbb{N}^{n}$ and $z \in \mathbb{R}^{n}$

- $\partial^{\alpha} / \partial z^{\alpha}=\partial^{\alpha_{1}} / \partial z_{1}^{\alpha_{1}} \ldots \partial^{\alpha_{n}} / \partial z_{n}^{\alpha_{n}}$, where $\alpha \in \mathbb{N}$ and $\left(z_{1}, \ldots, z_{n}\right)$ are coordinates.

Throughout the paper, the functions and vector fields are supposed to be analytic.

\subsection{Homogeneity}

We recall here the definition of homogeneity and direct properties, see $[13,14]$ for more details.

Definition 2.1. The function

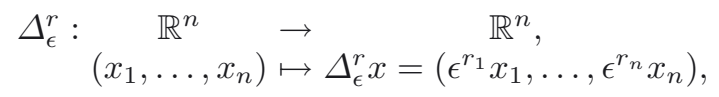

is called a dilation with respect to the weights $r=\left(r_{1}, \ldots, r_{n}\right) \in \mathbb{N}^{n}$, where $\epsilon>0$. 
Definition 2.2. A real valued function $h: \mathbb{R}^{n} \rightarrow \mathbb{R}$ is said to be homogeneous of degree $d$ with respect to the dilation $\Delta_{\epsilon}^{r}$ if

$$
h\left(\Delta_{\epsilon}^{r} x\right)=\epsilon^{d} h(x), \quad \forall x \in \mathbb{R}^{n},
$$

for all $\epsilon>0$. Equivalently, we say that $h$ is homogeneous of degree $d$ with respect to the weights $\left(r_{1}, \ldots, r_{n}\right)$.

Example 2.3. The function $h\left(x_{1}, x_{2}\right)=x_{1} x_{2}^{2}+x_{1}^{2}$ is homogeneous of degree 4 with respect to the weights $\left(r_{1}, r_{2}\right)=(2,1)$.

Definition 2.4. A vector field $f: \mathbb{R}^{n} \rightarrow \mathbb{R}^{n}$ is said to be homogeneous of degree $d$ with respect to the dilation $\Delta_{\epsilon}^{r}$ if

$$
f\left(\Delta_{\epsilon}^{r} x\right)=\epsilon^{d} \Delta_{\epsilon}^{r} f(x), \quad \forall x \in \mathbb{R}^{n},
$$

for all $\epsilon>0$.

Example 2.5. The vector field $f\left(x_{1}, x_{2}\right)=\left(x_{1} x_{2}^{2}\right) \frac{\partial}{\partial x_{1}}+\left(x_{1} x_{2}\right) \frac{\partial}{\partial x_{2}}$ is homogeneous of degree 2 with respect to the weights $\left(r_{1}, r_{2}\right)=(2,1)$.

The following lemma shows that the Lie derivative and the Lie bracket are compatible with the notion of homogeneity.

Lemma 2.6. The following results hold true:

(a) if $h_{1}, h_{2}: \mathbb{R}^{n} \rightarrow \mathbb{R}$ are homogeneous functions of degree $d_{1}$ and $d_{2}$ respectively, with respect to $\Delta_{\epsilon}^{r}$, then their product $h_{1} h_{2}$ is homogeneous of degree $d_{1}+d_{2}$ with respect to $\Delta_{\epsilon}^{r}$;

(b) if $h: \mathbb{R}^{n} \rightarrow \mathbb{R}$ is an homogeneous function of degree $d$ with respect to the dilation $\Delta_{\epsilon}^{r}$, then the partial derivative $\frac{\partial h}{\partial x_{i}}$ of $h$ relative to the ith coordinate is homogeneous of degree $d-r_{i}$ with respect to $\Delta_{\epsilon}^{r}$;

(c) if $f_{1}, f_{2}: \mathbb{R}^{n} \rightarrow \mathbb{R}^{n}$ are homogeneous vector fields of degree $d_{1}$ and $d_{2}$ respectively, with respect to $\Delta_{\epsilon}^{r}$, then the Lie bracket $\left[f_{1}, f_{2}\right]$ of $f_{1}, f_{2}$ is a homogeneous vector field of degree $d_{1}+d_{2}$ with respect to $\Delta_{\epsilon}^{r}$;

(d) if $h: \mathbb{R}^{n} \rightarrow \mathbb{R}$ and $f: \mathbb{R}^{n} \rightarrow \mathbb{R}^{n}$ are a function and a vector field homogeneous of degree $d_{1}$ and $d_{2}$ respectively, with respect to the dilation $\Delta_{\epsilon}^{r}$, then the Lie derivative $\mathcal{L}_{f} h$ of $h$ along the vector field $f$ is a homogeneous function of degree $d_{1}+d_{2}$ with respect to $\Delta_{\epsilon}^{r}$.

Proof. (a), (b) are given by property 2 and (c) by property 1 in [13]. A direct computation gives (d).

\subsection{Order induced by homogeneity}

Let $h: \mathbb{R}^{n} \rightarrow \mathbb{R}$ and $f: \mathbb{R}^{n} \rightarrow \mathbb{R}^{n}$ be a function and a vector field, respectively. Let $\Delta_{\epsilon}^{r}$ be a dilation with nonnegative integer weights. Then $h$ and $f$ admit an homogeneous expansion of the following form

$$
\begin{aligned}
& h(x)=\sum_{l=0}^{+\infty} h_{l}(x), \\
& f(x)=\sum_{l=-\max _{i}\left\{r_{i}\right\}}^{+\infty} f_{l}(x),
\end{aligned}
$$

where each $h_{l}: \mathbb{R}^{n} \rightarrow \mathbb{R}$ and $f_{l}: \mathbb{R}^{n} \rightarrow \mathbb{R}^{n}$ are homogeneous function and vector field, respectively, of degree $l$ with respect to $\Delta_{\epsilon}^{r}$. Indeed, $h$ an $f$ are analytic, therefore an expansion in polynomial functions exists. Since the weights are nonnegative integer, each polynomial function is homogeneous of a certain degree. We can switch the terms of this series, because it is absolutely convergent, in order to obtain the homogeneous expansion.

Definition 2.7. An analytic function $h: \mathbb{R}^{n} \rightarrow \mathbb{R}$ (respectively, an analytic vector field $f: \mathbb{R}^{n} \rightarrow \mathbb{R}^{n}$ ) is said to be of order $o(h)$ (respectively $o(f)$ ) greater or equal to $m \in \mathbb{Z}$ if all the terms of degree $d \leq m-1$ in the homogeneous expansion vanish. 
Example 2.8. The vector field $f(x)=\left(x_{2}+x_{1}+x_{2} x_{1}\right) \frac{\partial}{\partial x_{1}}+\left(x_{1}^{3}+x_{1} x_{2}+x_{2}^{2}\right) \frac{\partial}{\partial x_{2}}$ with the weights $\left(r_{1}, r_{2}\right)=$ $(1,2)$, admits the following homogeneous expansion

$$
f(x)=\sum_{l=-2}^{2} f_{l}(x),
$$

with $f_{-2}(x)=0, f_{-1}(x)=0, f_{0}(x)=\left(x_{1}\right) \frac{\partial}{\partial x_{1}}, f_{1}(x)=\left(x_{2}\right) \frac{\partial}{\partial x_{1}}+\left(x_{1}^{3}+x_{1} x_{2}\right) \frac{\partial}{\partial x_{2}}, f_{2}(x)=\left(x_{1} x_{2}\right) \frac{\partial}{\partial x_{1}}+\left(x_{2}^{2}\right) \frac{\partial}{\partial x_{2}}$. Furthermore $f$ is of order $o(f) \geq 0$.

\section{Construction of An homogeneous APPROXimation For the ObSERVABility PROBLEM}

Consider the following system

$$
\left\{\begin{array}{l}
\dot{x}=f(x), \quad x \in \mathbb{R}^{n}, \\
y=h(x)=\left(h_{1}(x), \ldots, h_{p}(x)\right), \quad y \in \mathbb{R}^{p},
\end{array}\right.
$$

where $x$ is the state and $y$ is the measured output. Functions $f$ and $h$ are supposed to be analytic and to satisfy $f(0)=0$ and $h(0)=0$. We assume that system (3.1) fulfills an observability rank condition, i.e.

$$
\operatorname{dim}(\mathrm{d} \mathcal{O}(0))=n,
$$

where

$$
\mathrm{d} \mathcal{O}=\{\mathrm{d} \gamma, \gamma \in \mathcal{O}\}
$$

and

$$
\mathcal{O}=\operatorname{span}\left\{\mathcal{L}_{f}^{i} h_{j}, i \in \mathbb{N}, j=1, \ldots, p\right\} .
$$

The space $\mathcal{O}$ is called the observability space of system (3.1).

The construction of the approximation is divided into two parts. First, we construct a change of coordinates $z=\varphi^{-1}(x)$. Then, we show that these new coordinates allow us to define an approximation which keeps observability properties unchanged at the origin.

\subsection{Construction of new coordinates}

The construction of the change of coordinates is split into four part

(1) definition of a flag on $\mathrm{d} \mathcal{O}$;

(2) definition of a basis of 1-forms for the flag, at the origin;

(3) construction of a dual basis of vector fields;

(4) definition of the change of coordinates.

\subsubsection{Definition of the flag}

In order to define an approximation which keeps the informations of the observability space at the origin of the original system, we need to put a structure on this space. This structure has to describe the action of the vector field $f$ on the output function, performed by the Lie derivative. Therefore, we consider the following flag

$$
L_{j}=\mathrm{d}\left(\operatorname{span}\left\{\mathcal{L}_{f}^{i} h_{k}: 0 \leq i \leq j-1, k=1, \ldots, p\right\}\right) .
$$

The flag $\left\{L_{j}\right\}_{j \geq 1}$ has the following properties

- $L_{j} \subset L_{j+1}, \quad j \geq 1$;

- $\mathcal{L}_{f} L_{j} \subset L_{j+1}, \quad j \geq 1$; 
- $\mathrm{d} \mathcal{O}=\cup_{j \geq 1} L_{j}$.

Remark 3.1. The subsets $L_{j}, j \geq 1$, contain only exact 1 -forms because of their construction. Hence, the Lie derivative $\mathcal{L}_{f} L_{j}$ of the subspace of 1 -forms $L_{j}$ is defined via the Cartan formula $\mathcal{L}_{f} d h=d \mathcal{L}_{f} h$, where $h: \mathbb{R}^{n} \rightarrow \mathbb{R}$.

\subsubsection{Definition of the basis}

Now that a structure on the space of observability has been defined, we need a related basis. Let us define first

$$
\begin{aligned}
n_{0} & =0, \\
n_{j} & =\operatorname{dim} L_{j}(0), \quad j \geq 1,
\end{aligned}
$$

and

$$
d=\min \left\{k: \operatorname{dim} L_{k}(0)=n\right\},
$$

where the number $d$ is a finite integer because of (3.2). We define next a basis $\left(\bar{\omega}_{i}\right)_{1 \leq i \leq n}$, following a recursive procedure:

- pick $\bar{\omega}_{1}, \ldots, \bar{\omega}_{n_{1}}$ in $L_{1}$ such that $\bar{\omega}_{1}(0), \ldots, \bar{\omega}_{n_{1}}(0)$ is a basis of $L_{1}(0)$;

- pick $\bar{\omega}_{n_{1}+1}, \ldots, \bar{\omega}_{n_{2}}$ in $L_{2}$ such that $\bar{\omega}_{1}(0), \ldots, \bar{\omega}_{n_{2}}(0)$ is a basis of $L_{2}(0)$,

- pick $\bar{\omega}_{n_{d-1}+1}, \ldots, \bar{\omega}_{n_{d}}$ in $L_{d}$ such that $\bar{\omega}_{1}(0), \ldots, \bar{\omega}_{n_{d}}(0)$ is a basis of $L_{d}(0)$.

Remark 3.2. By the definition of $d \mathcal{O}$, each $\bar{\omega}_{i}, 1 \leq i \leq n$ is exact, then we can associate a unique real function $\bar{h}_{i}: \mathbb{R}^{n} \rightarrow \mathbb{R}$ to $\bar{\omega}_{i}$ by

$$
\begin{aligned}
\mathrm{d} \bar{h}_{i} & =\bar{\omega}_{i}, \\
\bar{h}_{i}(0) & =0 .
\end{aligned}
$$

\subsubsection{Construction of the dual basis of vector fields}

The delicate part for the construction of the change of coordinates is to find a dual basis of vector fields with the required properties. We proceed as follow, first we state what properties are needed for the dual basis and then, we show that there always exists such a basis.

We define a basis of vector fields at the origin, of $\mathbb{R}^{n}$, as a set of $n$ vector fields $\bar{g}_{1}, \ldots, \bar{g}_{n}$ defined on a neighborhood $\mathcal{U}$ of the origin and such that $\bar{g}_{1}(0), \ldots, \bar{g}_{n}(0)$ are independent.

Let $V$ be the Lie algebra generated by $\bar{g}_{1}, \ldots, \bar{g}_{n}$ and $f$, and $\left(V_{j}\right)_{j \geq 0}$ the increasing sequence of subspaces of $V$, defined by

$$
V_{j}=\left\{g \in V: \bar{\omega}_{i}(g)=0,1 \leq i \leq n_{d-j}\right\} .
$$

A dual basis adapted to the flag (3.5) is defined as a basis $\bar{g}_{1}, \ldots, \bar{g}_{n}$ of vector fields at the origin which verify the following assumption:

\section{Assumption 3.3.}

(i) $V_{j} \subset V_{j+1}$;

(ii) $\left[V_{j}, V_{k}\right] \subset V_{j+k}$;

(iii) $\cup_{0 \leq j \leq d} V_{j}=V$; 


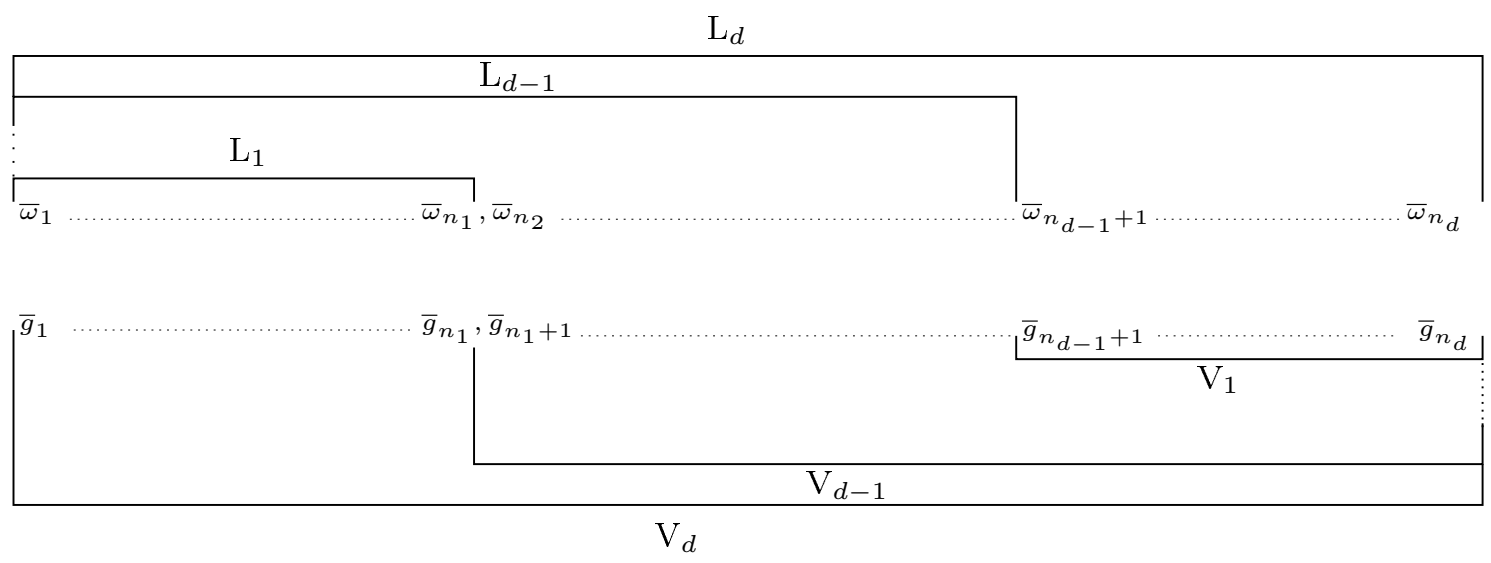

FiguRe 1.

(iv) For all $i=1, \ldots, n$

$$
\left(D \bar{h}_{i}\right)(0)=0, \quad \forall D \in B_{d-j} ;
$$

where $j \in\{1, \ldots, d\}$ is such that $n_{j-1}+1 \leq i \leq n_{j}$, and

$$
\begin{aligned}
B_{d-j-1} & =\operatorname{span}\left\{\mathcal{L}_{g_{1}} \circ \cdots \circ \mathcal{L}_{g_{k}} \mid k \in \mathbb{N},\left\{g_{1}, \ldots, g_{k}\right\} \subset\left\{\bar{g}_{1}, \ldots, \bar{g}_{n}\right\} \text { and } \sum_{l=1}^{k} w\left(g_{l}\right) \leq d-j-1\right\}, \\
w(g) & =\min \left\{k: g \in V_{k}\right\} .
\end{aligned}
$$

(v) For $k=1, \ldots, n$ and $j \in\{1, \ldots, d\}$ verifying $n_{j-1}+1 \leq k \leq n_{j}$, the following equality holds true

$$
\left(\bar{\omega}_{i}\left(\bar{g}_{k}\right)\right)(0)=0, \quad i=1, \ldots, n_{j-1} .
$$

Remark 3.4. Conditions (i)-(iii) are the same as in [13] and are used to obtain the approximation of the vector field. Condition (iv) is for the output function approximation. And the last condition ensures that the basis of vector fields are ordered with respect to $\left(V_{j}\right)$ as illustrated in Figure 1.

Now, we exhibit a vector fields basis satisfying Assumption 3.3. Consider the matrix $M(x)$ for which the columns are the components of the 1 -forms $\bar{\omega}_{1}, \ldots, \bar{\omega}_{n}$, i.e.

$$
M_{i j}(x)=\bar{\omega}_{j}^{i}(x), \quad \bar{\omega}_{j}(x)=\sum_{k=1}^{n} \bar{\omega}_{j}^{k}(x) \mathrm{d} x_{k}, \quad x \in \mathbb{R}^{n}, 1 \leq i, j \leq n .
$$

Since the observability rank condition is fulfilled at the origin, the matrix $M$ is invertible on a neighborhood $\mathcal{U}$ of the origin. Hence, there exist analytics vector fields $\tilde{g}_{1}, \ldots, \tilde{g}_{n}$ defined on $\mathcal{U}$, such that

$$
\bar{\omega}_{i}\left(\tilde{g}_{j}\right)(x)=\delta_{i}^{j}, \quad \forall x \in \mathcal{U} .
$$

Let $i=1, \ldots, n$, define

$$
\bar{g}_{i}(x)=\left(\tilde{g}_{n-i+1}\right)_{(j+2)}(x), \quad \forall x \in \mathcal{U},
$$

where $j \in\{1, \ldots, d\}$ is such that $n_{j-1} \leq n-j+1 \leq n_{j}$. Then we have the following result. 
Proposition 3.5. The dual basis defined by equation (3.13) verifies Assumption 3.3.

Remark 3.6. Another possible dual basis is given by

$$
\bar{g}_{i}=\tilde{g}_{n-i+1}, \quad i=1, \ldots, n .
$$

But as demonstrated by Proposition 3.5, we do not need to take an exact dual basis. Furthermore, this would make the computations for the change of coordinates unnecessarily complicated, or even impossible.

\subsubsection{Definition of the change of coordinates}

Consider a basis of vector fields at the origin $\bar{g}_{1}, \ldots, \bar{g}_{n}$ verifying Assumption 3.3. Then, we can define new coordinates $z=\varphi^{-1}(x), \varphi: \mathbb{R}^{n} \rightarrow \mathbb{R}^{n}$, by

$$
\varphi\left(z_{1}, \ldots, z_{n}\right)=\exp \left(z_{n} \bar{g}_{n}\right) \circ \cdots \circ \exp \left(z_{1} \bar{g}_{1}\right)(0)
$$

We call $z$, coordinates adapted to the flag (3.5). In the coordinates $z$ defined by (3.15), the system (3.1) becomes

$$
\left\{\begin{array}{l}
\dot{z}=\frac{\partial \varphi^{-1}}{\partial x}(\varphi(z)) \cdot f(\varphi(z)):=F(z) \\
y=h(\varphi(z)):=H(z)=\left(H_{1},(z), \ldots, H_{p}(z)\right)
\end{array}\right.
$$

similarly, we denote

$$
\begin{aligned}
& \bar{H}_{i}(z):=\bar{h}_{i}(\varphi(z)), \quad i=1, \ldots n, \\
& \bar{G}_{i}(z)=\frac{\partial \varphi^{-1}}{\partial x}(\varphi(z)) \cdot \bar{g}_{i}(\varphi(z)), \quad i=1, \ldots, n,
\end{aligned}
$$

and we have the following result:

Proposition 3.7 ([13], Prop. 2.1). In the new coordinates defined by (3.15), we have $\bar{G}_{i}(0)=\partial / \partial z_{i}, i=$ $1, \ldots, n$.

\subsection{Approximation design for the observability}

We now investigate the properties of the system (3.1) in the new coordinates defined by (3.15). This will allow us to define an homogeneous approximation which contains the informations of the observability space at the origin.

\subsubsection{Definition of the approximation}

We first define the weights $\left(r_{1}, \ldots, r_{n}\right)$ adapted to the filtration $\left(L_{j}\right)_{j \geq 1}$ at the origin by

$$
r_{i}=d-j, \quad n_{j}+1 \leq i \leq n_{j+1}, \quad j=0, \ldots, d-1 .
$$

The following main result is fundamental for our study, indeed, it shows that the order of $\bar{H}_{1}, \ldots \bar{H}_{n}$ and $F$ are suited to the definition of an homogeneous approximation with respect to the weights defined by (3.19).

Theorem 3.8. Let $F$ be given by (3.16) and $\bar{H}_{1}, \ldots, \bar{H}_{n}$ by equation (3.17). The order of $F$ and $\bar{H}_{1}, \ldots, \bar{H}_{n}$ with respect to the weights (3.19) verify:

- $o\left(\bar{H}_{i}\right) \geq d-j$, where $j \in\{1, \ldots, d\}$ is such that $n_{j-1}+1 \leq i \leq n_{j}$;

- $o(F) \geq-1$.

Remark 3.9. We have obtained properties on the order of the functions $\left(\bar{H}_{i}\right)_{i=1, \ldots, n}$. Thus, to define an approximation of the system (3.16), we need to have the same property to apply to the output function. That is why, in the following, we assume that $d h_{1}(0), \ldots, d h_{p}(0)$ are independent and that $\bar{h}_{1}=h_{1}, \ldots \bar{h}_{p}=h_{p}$. One can notice that $n_{1}=p$. 
The one-forms $\mathrm{d} h_{1}, \ldots, \mathrm{d} h_{p}$ associated to the output function of system (3.1) belong to $L_{1}$ by construction. Hence, applying Theorem 3.8, the homogeneous expansion of $F$ and $H_{i}, i=1, \ldots, p$ (given by (3.12)), with respect to the weights $\left(r_{1}, \ldots, r_{n}\right)$ can be written as

$$
\begin{aligned}
F(z) & =\sum_{l=-1}^{+\infty} F_{l}(z), \\
H_{i}(z) & =\sum_{l=d-1=r_{1}}^{+\infty} H_{i}^{l}(z),
\end{aligned}
$$

where for each $l, F_{l}$ and $H_{i}^{l}$ are homogeneous of degree $l$ with respect to the weights (3.19).

Definition 3.10. The approximation of system (3.16) with respect to the flag $\left(L_{j}\right)_{j \geq 1}$ is defined as

$$
\begin{aligned}
\tilde{H}_{i}(z) & =H_{i}^{d-1}(z), \\
\tilde{F}(z) & =F_{-1}(z) .
\end{aligned}
$$

Remark 3.11. The approximation simply consists in taking the first term in the homogeneous approximation with respect to the given weights.

\subsubsection{Properties of the approximation}

The following result states, in a precise way, the correspondence between the observability space of the original system (3.1) and its approximating system

$$
\left\{\begin{array}{l}
\dot{z}=\tilde{F}(z), \quad z \in \mathbb{R}^{n}, \\
y=\tilde{H}(z), \quad y \in \mathbb{R}^{p} .
\end{array}\right.
$$

Theorem 3.12. Let $\omega \in L_{1}$ and let $\tilde{\omega}$ be the corresponding one-forms given by the approximating system (3.24). Let $k \in\{0, \ldots, d-1\}$, then if we denote $\mathcal{L}_{F}^{k} \omega(z)=\sum_{i=1}^{n} a_{i}(z) \mathrm{d} z_{i}$ and $\mathcal{L}_{\tilde{F}}^{k} \tilde{\omega}(z)=\sum_{i=1}^{n} b_{i}(z) \mathrm{d} z_{i}$, we have

$$
\begin{aligned}
& a_{i}(0)=b_{i}(0), \quad n_{k}+1 \leq i \leq n_{k+1}, \\
& a_{i}(0)=b_{i}(0)=0, n_{k+1}+1 \leq i \leq n .
\end{aligned}
$$

Let $\left(\tilde{L}_{j}\right)_{j \geq 1}$ be the flag associated to the approximating system (3.24), the following corollary is a direct consequence of Theorem 3.12 and hence will not be proved.

Corollary 3.13. For each $j=1, \ldots, d$, we have the following equality

$$
L_{j}(0)=\tilde{L}_{j}(0) .
$$

\section{LOCAL OBSERVER DESIGN}

In this section, we present the construction of a local observer, using the approximation developed in the previous section. We consider the case of a system with an output of dimension 1 to avoid unnecessary complicated notations, but the extension to the multi-output case follows the same lines. Let us consider the system

$$
\begin{cases}\dot{x}=f(x), & x \in \mathbb{R}^{n}, \\ y=h(x), & y \in \mathbb{R},\end{cases}
$$

where $f$ and $h$ are analytics. We assume that

$$
\operatorname{dim}\left(\operatorname{span}\left\{\mathrm{d} \mathcal{L}_{f}^{k} h(0), k=0, \ldots, n-1\right\}\right)=n .
$$

Hence system (4.1) verifies the observability rank condition. 
According to the previous section, there exists new coordinates $z$ defined by $z=\varphi^{-1}(x)$ and weights $r_{i}=n-i$, $i=1, \ldots, n$, such that, in the new coordinates, $F(z)=\left(\partial \varphi^{-1} / \partial x\right)(\varphi(z)) \cdot f(\varphi(z))$ and $H(z)=h(\varphi(z))$ are of order greater or equal to -1 and $r_{1}=n-1$ respectively. The approximating system is then given by

$$
\left\{\begin{array}{l}
\dot{z}=\tilde{F}(z), \\
y=\tilde{H}(z),
\end{array}\right.
$$

where $\tilde{F}(z)=F_{-1}(z)$ and $\tilde{H}(z)=H^{r_{1}}(z)$ are the first terms of the homogeneous expansion of $F$ and $H$ respectively, with respect to weights $r_{i}=n-i, i=1, \ldots, n$.

In order to construct an observer for system (4.1), we first design an observer for system (4.3). We use the high gain observer presented in [9] after having transformed the system (4.3) into an observability canonical form. The observer for the original system is then obtained simply by replacing $\tilde{f}$ and $\tilde{h}$ by $f$ and $h$.

The second change of coordinates is given by

$$
\xi=\Phi_{h}(z)=\left(\begin{array}{c}
\tilde{H}(z) \\
\mathcal{L}_{\tilde{F}} \tilde{H}(z) \\
\vdots \\
\mathcal{L}_{\tilde{F}}^{n-1} \tilde{H}(z)
\end{array}\right)
$$

In the new coordinates $\xi$, system (4.3) is in the following observability canonical form

$$
\left\{\begin{array}{l}
\dot{\xi}=A \xi, \\
y=C \xi,
\end{array}\right.
$$

where $A_{i j}=\delta_{i}^{j-1}, 1 \leq i, j \leq n$ and $C=\left[\begin{array}{lll}1 & 0 \ldots 0\end{array}\right]$. An observer is thus given by

$$
\dot{\hat{\xi}}=A \hat{\xi}-K(\theta) C(\hat{\xi}-\xi),
$$

where the gain $K(\theta)$ is given by

$$
K(\theta):=\left[\begin{array}{c}
\theta C_{n}^{1} \\
\theta^{2} C_{n}^{2} \\
\vdots \\
\theta^{n} C_{n}^{n}
\end{array}\right], \quad C_{n}^{p}=\frac{n !}{(n-p) ! p !}, \theta \in \mathbb{R} .
$$

In the original coordinates $x$, the gain $\bar{K}_{h}(\theta, \hat{x})$ reads

$$
\bar{K}_{h}(\theta, \hat{x})=\frac{\partial\left(\varphi \circ \Phi_{h}^{-1}\right)}{\partial \xi}\left(\Phi_{h} \circ \varphi^{-1}(\hat{x})\right) K(\theta),
$$

and the observer for the original system (4.1)

$$
\dot{\hat{x}}=f(\hat{x})-\bar{K}_{h}(\theta, \hat{x})(h(\hat{x})-h(x)) .
$$

We have the following result of convergence for the observer (4.9).

Proposition 4.1. Assume that system (4.1) verifies property (4.2). Then, there exists $\theta^{*}>0$ such that for all $\theta>\theta^{*}$, there exist $\mathcal{V}_{1}(\theta)$ and $\mathcal{V}_{2}(\theta)$, two neighborhoods of the origin, such that:

if $\mathcal{V}_{1}$ positively invariant under system (4.1) then the observer (4.8)-(4.9) is convergent on $\mathcal{V}_{2}$. 
Remark 4.2. Proposition 4.1 states that for every $\theta>\theta^{*}$, there exists a set in which the approximation is valid. Thus if the solutions of system (4.1) stay in this set, the observer is locally convergent.

The following proposition is similar to Proposition 4.1 but with different assumptions.

Proposition 4.3. If system (4.1) verifies property (4.2) and if its origin is Lyapunov stable, then there exists a real number $\theta^{*}>0$, such that for all $\theta>\theta^{*}$, there exists a neighborhood of the origin $\mathcal{U}(\theta)$ such that observer (4.8)-(4.9) is convergent on $\mathcal{U}$.

Remark 4.4. Proposition 4.1 and 4.3 can be seen as an application of Theorem 1 in [26], which is derived from the center manifold theory. But we propose here an original proof, derived from Lyapunov theory, which give the possibility to obtain an analytic estimation of the domain of convergence.

\section{EXAMPLe}

In this section, we apply the methodology developed in this paper on a particular example and we compare it to the observer given by the linear approximation.

\subsection{System}

The considered system is

$$
\left\{\begin{array}{l}
\dot{x}_{1}=-x_{1}+x_{2}+x_{2}^{2}-x_{3}^{3}-x_{2}^{3}, \\
\dot{x}_{2}=x_{3}-x_{2}+x_{1}-x_{3}^{3}-x_{1}^{5}, \\
\dot{x}_{3}=-x_{3}^{5}-x_{2}^{3} \\
y=h(x)=x_{2}+x_{1}^{2}+x_{1} x_{3}+x_{3}^{2} .
\end{array}\right.
$$

This system fulfill the observability rank condition, since

$$
\begin{aligned}
\mathrm{d} h(0) & =\mathrm{d} x_{2}, \\
\mathrm{~d} \mathcal{L}_{f} h(0) & =\mathrm{d} x_{3}-\mathrm{d} x_{2}+\mathrm{d} x_{1}, \\
\mathrm{~d} \mathcal{L}_{f}^{2} h(0) & =-2 \mathrm{~d} x_{1}+2 \mathrm{~d} x_{2}-\mathrm{d} x_{3} .
\end{aligned}
$$

Thus $\operatorname{dim} \mathrm{d} \mathcal{O}(0)=3$.

\subsection{Observer design by homogeneous approximation}

We can apply our method:

- Computation of the first change of coordinates:

A basis for the flag is given by

$$
\begin{aligned}
\mathrm{d} \bar{h}_{1} & =\mathrm{d} h, \\
\mathrm{~d} \bar{h}_{2} & =\mathrm{d} \mathcal{L}_{f} h, \\
\mathrm{~d} \bar{h}_{3} & =\mathrm{d} \mathcal{L}_{f}^{2} h .
\end{aligned}
$$

It is straightforward to check that the following dual basis fulfill the requirements of Assumption 3.3

$$
\begin{aligned}
\bar{g}_{1}(x) & =\frac{\partial}{\partial x_{2}} \\
\bar{g}_{2}(x) & =\frac{\partial}{\partial x_{1}} \\
\bar{g}_{3}(x) & =-\frac{\partial}{\partial x_{1}}+2 x_{1} \frac{\partial}{\partial x_{2}}+\frac{\partial}{\partial x_{3}} .
\end{aligned}
$$


Then the diffeomorphism (3.15) is given by

$$
\varphi(z)=\left(\begin{array}{c}
-z_{3}+z_{2} \\
-z_{3}^{2}+z_{1}+2 z_{2} z_{3} \\
z_{3}
\end{array}\right), \quad \varphi^{-1}(x)=\left(\begin{array}{c}
x_{2}-2 x_{1} x_{3}-x_{3}^{2} \\
x_{1}+x_{3} \\
x_{3}
\end{array}\right) .
$$

Hence, we obtain the following approximation

$$
\left\{\begin{array}{l}
\dot{z}_{1}=-z_{3}^{2}+z_{2} \\
\dot{z}_{2}=z_{3} \\
\dot{z}_{3}=0 \\
y=z_{1}+z_{2} z_{3} .
\end{array}\right.
$$

- Construction of the second change of coordinates:

By definition

$$
\xi=\Phi_{h}(z)=\left(\begin{array}{c}
\tilde{H}(z) \\
\mathcal{L}_{\tilde{F}} \tilde{H}(z) \\
\mathcal{L}_{\tilde{F}}^{2} \tilde{H}(z)
\end{array}\right)
$$

Thus

$$
\Phi_{h}(z)=\left(\begin{array}{c}
z_{1}+z_{2} z_{3} \\
z_{2} \\
z_{3}
\end{array}\right), \quad \Phi_{h}^{-1}(\xi)=\left(\begin{array}{c}
\xi_{1}-\xi_{2} \xi_{3} \\
\xi_{2} \\
\xi_{3}
\end{array}\right)
$$

- Construction of the observer:

The change of coordinates from $x$ to $\xi$ is given by

$$
\xi=\Phi_{h} \circ \varphi^{-1}(x)=\left(\begin{array}{c}
x_{2}-x_{1} x_{3} \\
x_{1}+x_{3} \\
x_{3}
\end{array}\right), \quad x=\varphi \circ \Phi_{h}^{-1}(\xi)=\left(\begin{array}{c}
\xi_{2}-\xi_{3} \\
-\xi_{3}^{2}+\xi_{1}+\xi_{2} \xi_{3} \\
\xi_{3}
\end{array}\right) .
$$

Then

$$
\bar{K}_{h}(\theta, \hat{x})=\left(\begin{array}{c}
3 \theta-\theta^{3} \\
3 \theta+\theta^{3} \hat{x}_{1}+\left(3 \theta^{2}-\theta^{3}\right) \hat{x}_{3} \\
\theta^{3}
\end{array}\right),
$$

with $K(\theta)=\left[3 \theta, 3 \theta^{2}, \theta^{3}\right]^{T}$. The observer is given by

$$
\left\{\begin{aligned}
\dot{\hat{x}}_{1}= & -\hat{x}_{1}+\hat{x}_{2}+\hat{x}_{2}^{2}-\hat{x}_{3}^{3}-\hat{x}_{2}^{3} \\
& -\left(3 \theta-\theta^{3}\right)\left(\left(\hat{x}_{2}+\hat{x}_{1}^{2}+\hat{x}_{1} \hat{x}_{3}+\hat{x}_{3}^{2}\right)-\left(x_{2}+x_{1}^{2}+x_{1} x_{3}+x_{1}^{2}\right)\right), \\
\dot{\hat{x}}_{2}= & \hat{x}_{3}-\hat{x}_{2}+\hat{x}_{1}-\hat{x}_{3}^{3}-\hat{x}_{1}^{5} \\
& -\left(3 \theta+\theta^{3} \hat{x}_{1}+\left(3 \theta^{2}-\theta^{3}\right) \hat{x}_{3}\right)\left(\left(\hat{x}_{2}+\hat{x}_{1}^{2}+\hat{x}_{1} \hat{x}_{3}+\hat{x}_{3}^{2}\right)-\left(x_{2}+x_{1}^{2}+x_{1} x_{3}+x_{1}^{2}\right)\right), \\
\dot{\hat{x}}_{3}= & \hat{x}_{3}^{5}-\hat{x}_{2}^{3}-\left(\theta^{3}\right)\left(\left(\hat{x}_{2}+\hat{x}_{1}^{2}+\hat{x}_{1} \hat{x}_{3}+\hat{x}_{3}^{2}\right)-\left(x_{2}+x_{1}^{2}+x_{1} x_{3}+x_{1}^{2}\right)\right) .
\end{aligned}\right.
$$

\subsection{Observer design by linear approximation}

In order to compare our approach with the linear approximation, we apply the same method:

- Definition of the linear approximation

$$
\left\{\begin{array}{l}
\dot{x}_{1}=-x_{1}+x_{2}, \\
\dot{x}_{2}=x_{3}-x_{2}+x_{1}, \\
\dot{x}_{3}=0 \\
y=x_{2} .
\end{array}\right.
$$


- Computation of the change of coordinates

$$
\xi=\Phi_{l}(x)=\left(\begin{array}{c}
\tilde{h}_{l}(x) \\
\mathcal{L}_{\tilde{f}_{l}} \tilde{h}_{l}(x) \\
\mathcal{L}_{\tilde{f}_{l}}^{2} \tilde{h}_{l}(x)
\end{array}\right)=\left(\begin{array}{c}
x_{2} \\
x_{1}-x_{2}+x_{3} \\
-2 x_{1}+2 x_{2}-x_{3}
\end{array}\right), \quad \Phi_{l}^{-1}(\xi)=\left(\begin{array}{c}
\xi_{1}-\xi_{2}-\xi_{3} \\
\xi_{1} \\
2 \xi_{1}+\xi_{3}
\end{array}\right) .
$$

- Construction of the observer

$$
\bar{K}_{l}(\theta)=\frac{\partial \Phi_{l}^{-1}}{\partial \xi}\left(\Phi_{l}(\hat{x})\right) K(\theta)=\left(\begin{array}{c}
3 \theta-3 \theta^{2}-\theta^{3} \\
3 \theta \\
3 \theta^{2}+\theta^{3}
\end{array}\right) .
$$

with $K(\theta)=\left[3 \theta, 3 \theta^{2}, \theta^{3}\right]^{T}$. We obtain the following observer

$$
\left\{\begin{aligned}
\dot{\hat{x}}_{1}= & -\hat{x}_{1}+\hat{x}_{2}+\hat{x}_{2}^{2}-\hat{x}_{3}^{3}-\hat{x}_{2}^{3} \\
& -\left(3 \theta-3 \theta^{2}-\theta^{3}\right)\left(\left(\hat{x}_{2}+\hat{x}_{1}^{2}+\hat{x}_{1} \hat{x}_{3}+\hat{x}_{3}^{2}\right)-\left(x_{2}+x_{1}^{2}+x_{1} x_{3}+x_{3}^{2}\right)\right), \\
\dot{\hat{x}}_{2}= & \hat{x}_{3}-\hat{x}_{2}+\hat{x}_{1}-\hat{x}_{3}^{3}-\hat{x}_{1}^{5} \\
& -(3 \theta)\left(\left(\hat{x}_{2}+\hat{x}_{1}^{2}+\hat{x}_{1} \hat{x}_{3}+\hat{x}_{3}^{2}\right)-\left(x_{2}+x_{1}^{2}+x_{1} x_{3}+x_{3}^{2}\right)\right), \\
\dot{\hat{x}}_{3}= & \hat{x}_{3}^{5}-\hat{x}_{2}^{3} \\
& -\left(3 \theta^{2}+\theta^{3}\right)\left(\left(\hat{x}_{2}+\hat{x}_{1}^{2}+\hat{x}_{1} \hat{x}_{3}+\hat{x}_{3}^{2}\right)-\left(x_{2}+x_{1}^{2}+x_{1} x_{3}+x_{3}^{2}\right)\right) .
\end{aligned}\right.
$$

\subsection{Results of simulations}

We have computed an estimation of the domain of convergence of both observers by numerical means. More precisely, we have determined the larger $\delta>0$ such that $\mathcal{B}(0, \delta) \times \mathcal{B}(0, \delta)$ is in the domain of convergence $\mathcal{U}$ of the observer. For each $\delta$, we have run 100000 simulations where each corresponds to a couple of initial conditions taken randomly in $\mathcal{B}(0, \delta) \times \mathcal{B}(0, \delta)$. An initial condition is said to be into the domain of convergence if at time $t_{\text {simulation }}=30 \mathrm{~s}$ the error between the original system and the observer is less than $\epsilon_{\text {simulation }}=10^{-3}$. The results are given in (5.13).

\begin{tabular}{|c|c|c|}
\hline & $\delta$ observer by homogeneous approximation & $\delta$ observer by linear approximation \\
\hline$\theta=1$ & 0.56 & 0.3 \\
\hline$\theta=2$ & 0.3 & 0.2 \\
\hline
\end{tabular}

We illustrate the convergence of both observers for particular initial conditions. We consider the initial condition $x_{0}=[0.1,0.1,0.1]$ for the original system and $\hat{x}_{0}=[-0.2,0.1,0.05]$ for both observers. The results are given in Figure 2 for $\theta=1$ and in Figure 3 for $\theta=2$, where the original system is represented with solide lines while the observers are represented with dashed lines. We notice that the behavior of both observers is similar for $\theta=1$, but for $\theta=2$, as expected from the estimation of the domain of convergence, the homogeneous approximation observer is still convergent but not the linear one, which explode in finite time, indeed $\left\|x_{0}\right\|_{2} \approx$ 0.17 and $\left\|\hat{x}_{0}\right\|_{2} \approx 0.23$.

\section{Proofs}

\subsection{Proof of Proposition 3.5}

In order to prove Proposition 3.5, we need the following technical result:

Lemma 6.1. Let $g: \mathcal{U} \rightarrow \mathbb{R}$ be an analytic vector field and $\omega$ be an analytic one form defined on $\mathcal{U}$, where $\mathcal{U}$ is a neighborhood of the origin in $\mathbb{R}^{n}$, such that

$$
\omega(g)(x)=0, \quad \forall x \in \mathcal{U} .
$$



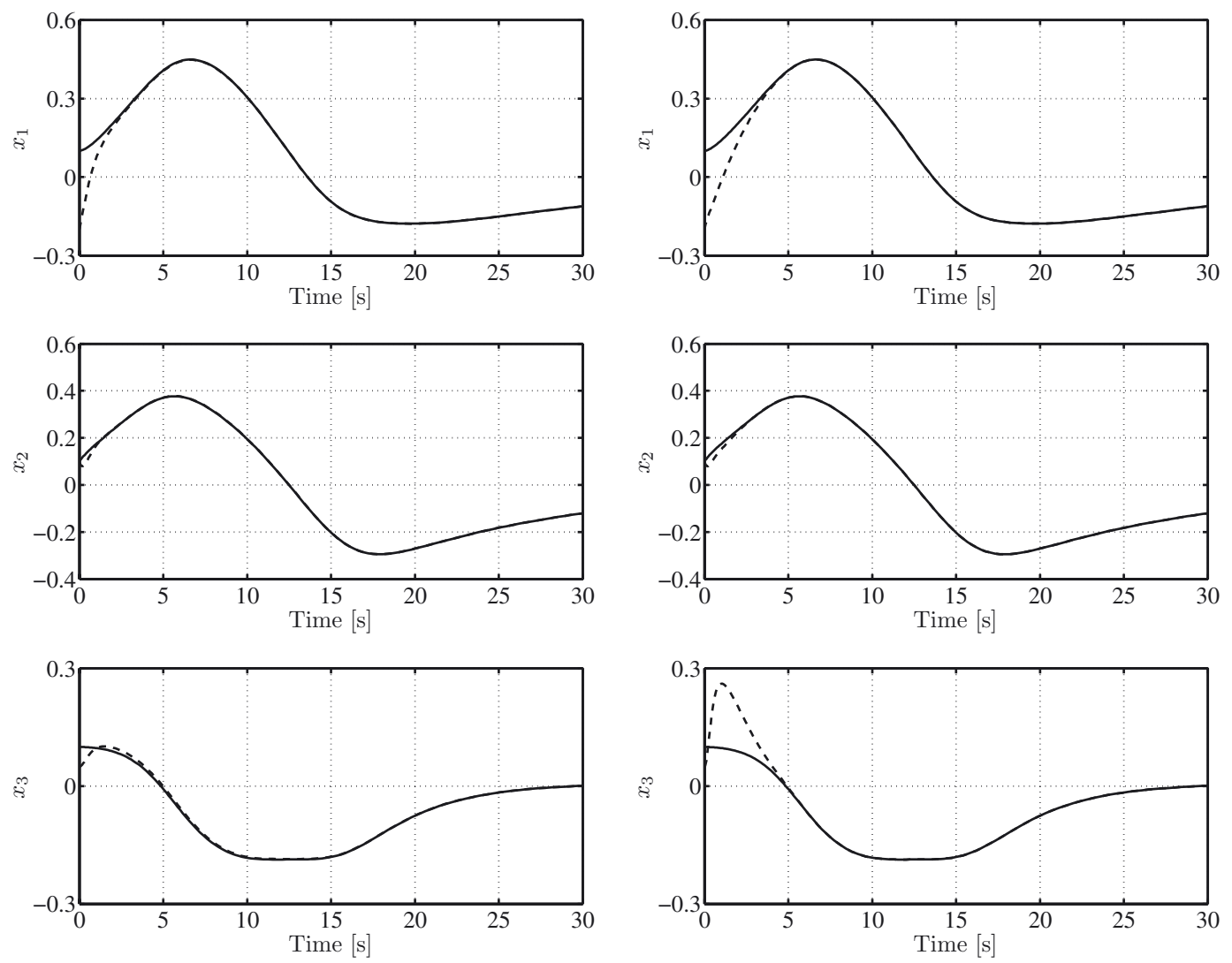

homogeneous approximation observer

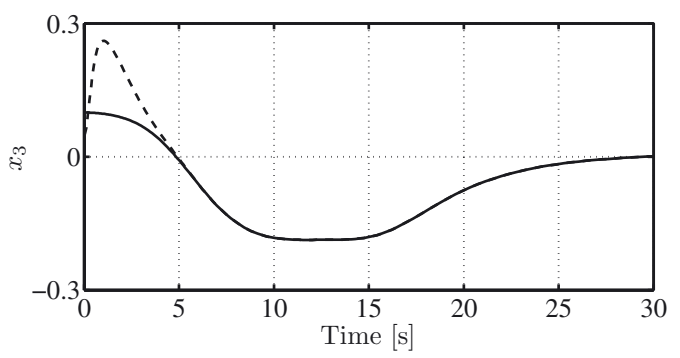

linear approximation observer

Figure 2. Original system (5.1) (solid line) and the two observers (dashed line) with a gain $\theta=1$.

If we denote $g_{(k)}$ the Taylor expansion of $g$ up to order $k$, then

$$
\left(\omega\left(g_{(k)}\right)\right)_{(l)}=0, \quad l=0, \ldots, k-1 .
$$

Proof of Lemma 6.1. Since $g$ and $\omega$ are analytics, we can write $g$ as followed:

$$
g(x)=\sum_{i=1}^{n}\left(\sum_{k=0}^{+\infty} g_{k}^{i}(x)\right) \frac{\partial}{\partial x_{i}}, \quad x \in \mathcal{U}
$$

where $g_{k}^{i}(x)$ is the $k$ th term in the Taylor expansion of the real valued function $g^{i}(x)$, and

$$
\omega(x)=\sum_{i=1}^{n}\left(\sum_{k=0}^{+\infty} \omega_{k}^{i}(x)\right) \mathrm{d} x_{i}, \quad x \in \mathcal{U}
$$



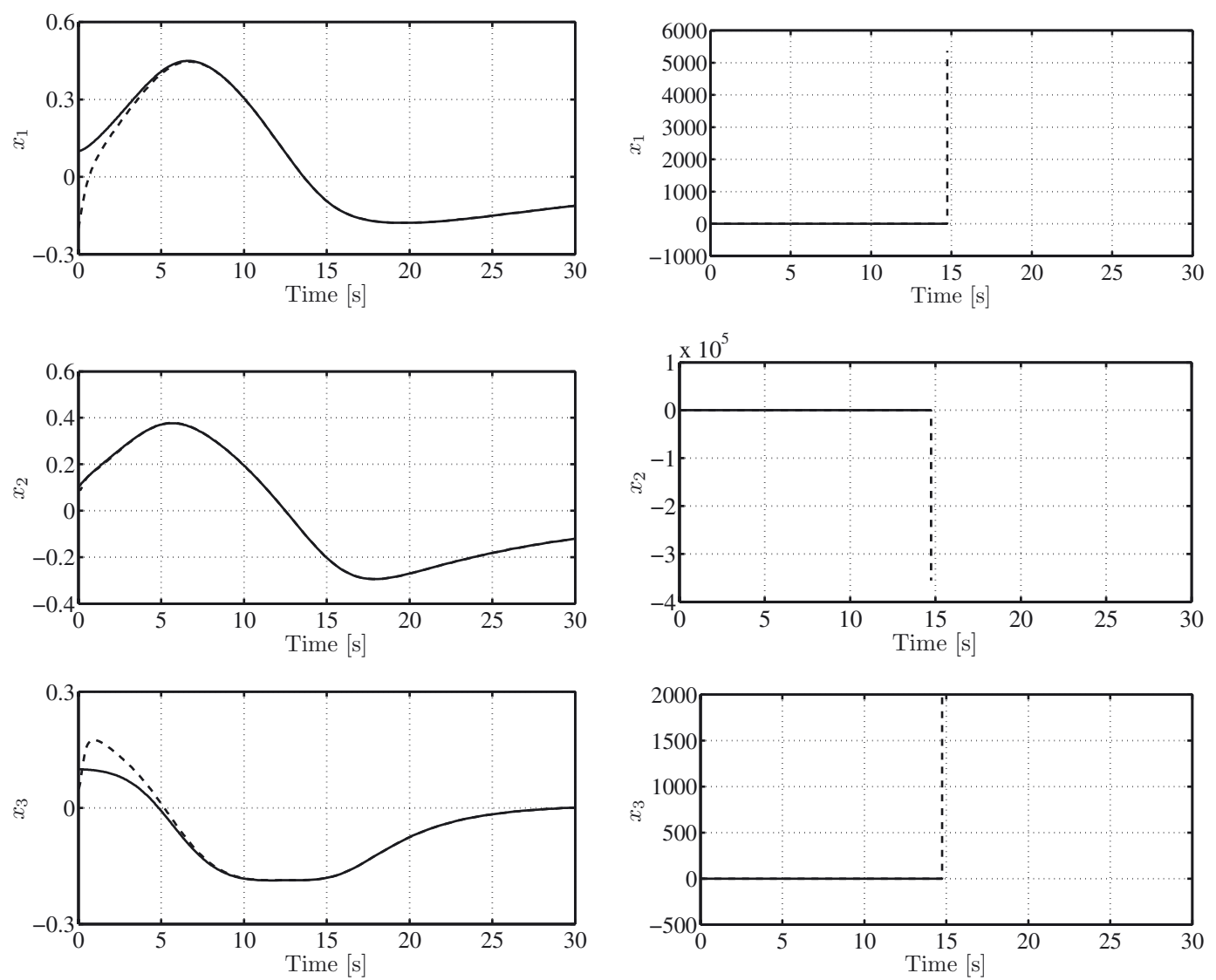

homogeneous approximation observer

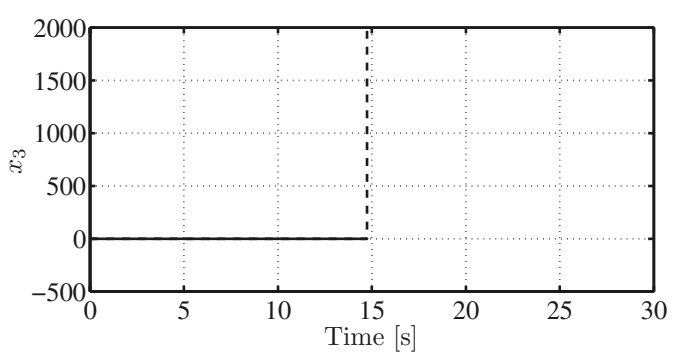

linear approximation observer

Figure 3. Original system (5.1) (solid line) and the two observers (dashed line) with a gain $\theta=2$.

where $\omega_{k}^{i}(x)$ is the $k$ th term in the Taylor expansion of $\omega^{i}$. Thus

$$
\begin{aligned}
\omega(g)(x) & =\sum_{i=1}^{n}\left(\sum_{k_{1}=0}^{+\infty} g_{k_{1}}^{i}(x)\right)\left(\sum_{k_{2}=0}^{+\infty} \omega_{k_{2}}^{i}(x)\right), \\
& =\sum_{i=1}^{n}\left(\sum_{l=0}^{+\infty} \sum_{m=0}^{l} g_{m}^{i}(x) \omega_{l-m}^{i}(x)\right) \\
& =\sum_{l=0}^{+\infty}\left(\sum_{i=1}^{n} \sum_{m=0}^{l} g_{m}^{i}(x) \omega_{l-m}^{i}(x)\right)
\end{aligned}
$$

is the Taylor expansion of the function $\omega(g)$. Since $\omega(g)$ is analytic and vanish in a neighborhood $\mathcal{U}$ of the origin, we necessarily have

$$
\sum_{i=1}^{n} \sum_{m=0}^{l} g_{m}^{i}(x) \omega_{l-m}^{i}(x)=0, \quad \forall x \in \mathcal{U}, \quad \forall l \in \mathbb{N} .
$$

Hence, if $g_{(k)}$ is taken instead of $g$, the first $(k-1)$ terms of the Taylor expansion of $\omega(g)$ vanish, i.e. $(\omega(g))_{(l)}=$ $0, l=0, \ldots, k-1$. 
Proof of Proposition 3.5.

\section{Points $(i)$ and $(v)$}

These properties are direct consequences of the definition of $\left(\bar{g}_{i}\right)_{1 \leq i \leq n}$ and $\left(V_{j}\right)_{j \geq 1}$.

\section{Point (iv)}

Let $i \in\{1, \ldots, n\}$, then there exists $j \in\{1, \ldots, d\}$ such that $n_{j-1}+1 \leq i \leq n_{j}$. We have to prove that for all $D \in B_{d-j}$

$$
D \bar{h}_{i}(0)=0 .
$$

Since $D$ is in $B_{d-j}$, we can write

$$
D=\mathcal{L}_{g_{1}} \ldots \mathcal{L}_{g_{k}}, \quad \text { with } \sum_{l=1}^{k} w\left(g_{l}\right) \leq d-j .
$$

Let $p=w\left(g_{k}\right)$, by definition of $B, g_{k} \in\left\{\bar{g}_{1}, \ldots, \bar{g}_{n-n_{d-p}}\right\}$. Apply Lemma 6.1 with equation (3.13), we get

$$
\left(\mathcal{L}_{g_{k}} \bar{h}_{i}\right)_{(l)}(0)=0, \quad 0 \leq l \leq d-p+1 .
$$

We have $k-1<d-p+1$, because $(k-1)+p \leq d-j \leq d$, hence

$$
D \bar{h}_{i}(0)=\mathcal{L}_{g_{1}} \ldots \mathcal{L}_{g_{k}} \bar{h}_{i}(0)=0 .
$$

\section{Point (ii)}

Since $V$ is spanned by $f$ and $\left\{\bar{g}_{1}, \ldots, \bar{g}_{n}\right\}$, we just have to show that, for a given $k \in \mathbb{N}$ and vector fields $g_{1}, \ldots, g_{k} \in\left\{\bar{g}_{1}, \ldots, \bar{g}_{n}, f\right\}$ verifying $\sum_{l=1}^{k} w\left(g_{l}\right)=j$, the following property is verified

$$
a d_{g_{1}} \ldots a d_{g_{k-1}} g_{k} \in V_{j}
$$

i.e., we have to prove that

$$
\bar{\omega}_{i}\left(a d_{g_{1}} \ldots a d_{g_{k-1}} g_{k}\right)(0)=0, \quad 1 \leq i \leq n_{d-j} .
$$

The left hand-side of equation (6.14) can be rewritten as

$$
\mathcal{L}_{a d_{g_{1}} \ldots a d_{g_{k-1}} g_{k}} \bar{h}_{i}(0) .
$$

For two vector fields $\tilde{f}, \tilde{g}: \mathcal{U} \rightarrow \mathbb{R}^{n}$ and a function $\tilde{h}: \mathcal{U} \rightarrow \mathbb{R}^{n}$, we have

$$
\mathcal{L}_{[\tilde{f}, \tilde{g}]} h=\mathcal{L}_{\tilde{f}} \mathcal{L}_{\tilde{g}} h-\mathcal{L}_{\tilde{g}} \mathcal{L}_{\tilde{f}} h .
$$

Hence (6.15) can be written as the sum of terms of the type $c \mathcal{L}_{g_{s_{1}}} \ldots \mathcal{L}_{g_{s_{k}}} \bar{h}_{i}$ with $\left\{s_{1}, \ldots, s_{k}\right\}=\{1, \ldots, k\}$ and $c= \pm 1$. There is two possible cases. First, assume that $g_{s_{k}}$ belongs to $\left\{\bar{g}_{1}, \ldots, \bar{g}_{n}\right\}$. In this case, the idea of the proof is the same that for property $(i v)$. Indeed, we have to prove that

$$
\mathcal{L}_{g_{s_{1}}} \ldots \mathcal{L}_{g_{s_{k}}} \bar{h}_{i}(0)=0
$$

where $1 \leq i \leq n_{d-j}$ and $\sum_{l=1}^{k} w\left(g_{l}\right) \leq j$. Take $\tilde{j}=d-j$, it comes $1 \leq i \leq n_{\tilde{j}}$ and $\sum_{l=1}^{k} w\left(g_{l}\right) \leq n_{d-\tilde{j}}$, hence equation (6.17) can be proven exactly in the same fashion than property $(\mathrm{iv})$.

The second case happens when $g_{s_{k}}=f$. In that case, we have

$$
\mathcal{L}_{g_{s_{1}}} \ldots \mathcal{L}_{g_{s_{k}}} \bar{h}_{i}=\mathcal{L}_{g_{s_{1}}} \ldots \mathcal{L}_{g_{s_{k-1}}} \circ \mathcal{L}_{f} \bar{h}_{i}
$$

Since $\mathcal{L}_{f} \bar{h}_{i}$ belong to $L_{d-j+1}$ and $\sum_{l=1}^{k-1} w\left(g_{l}\right) \leq d-j-1$, then, again, we check if $g_{s_{k-1}}$ is equal to $f$ or belong to $\left\{\bar{g}_{1}, \ldots, \bar{g}_{n}\right\}$. If there is only $f$ vector fields, then equation (6.15) holds true because $f(0)=0$ and $\bar{h}_{i}(0)=0$. This ends the proof. 


\subsection{Proof of Theorem 3.8}

Order of the vector field $f$.. Following the notations of [13], a basis adapted to the filtration $\left(V_{j}\right)_{j \geq 1}$ is given by $\bar{g}_{n}, \ldots, \bar{g}_{1}$. More specifically, $\bar{g}_{n_{d}}(0), \ldots, \bar{g}_{n_{d-1}+1}(0)$ is a basis of $V_{1}$ at zero, $\bar{g}_{n_{d}}(0), \ldots, \bar{g}_{n_{d-2}+1}(0)$ is a basis of $V_{2}$ at zero, etc. According to Theorem 2.1 in [13], in the the new coordinates $z$ defined by the inverse of the function

$$
\varphi(z)=\exp \left(z_{n} \bar{g}_{n}\right) \circ \cdots \circ \exp \left(z_{1} \bar{g}_{1}\right)(0)
$$

if $g \in V_{j}$, we have

$$
o(G) \geq-j, \quad \text { with respect to the weights } r_{i}=d-j, \quad n_{j-1}+1 \leq i \leq n_{j},
$$

where $G(z)=\left(\partial \varphi^{-1} / \partial x\right)(\varphi(z)) g(\varphi(z))$ is the vector field $g$ written in the new coordinates. The basis taken here is in reverse order (for the index) compared to the one in [13], it explains why the weights are reversed too.

In particular, the vector field $f$ belongs to $V_{1}$, since $\bar{\omega}_{i}(f)(0)=0$ for all $i=1, \ldots, n$. Applying (6.20), we obtain the result.

Order of the functions $\bar{H}_{i}, i=1, \ldots, n$. We need to prove the following statement

$$
\frac{\partial^{\alpha}}{\partial z^{\alpha}} \bar{H}_{i}(0)=0, \quad \forall \alpha \in \mathbb{N}^{n} \text { such that }\|\alpha\|_{r} \leq d-j-1 .
$$

By Assumption 3.3 (iv), we know that, in the $x$ coordinates,

$$
D \bar{h}_{i}(0)=0, \quad \forall D \in B_{d-j-1} .
$$

In the following, we will prove that (6.22) actually implies (6.21). First, we rewrite (6.22) in the new coordinates. Let us denote $\bar{G}_{1}, \ldots, \bar{G}_{n}$ for the vector fields $\bar{g}_{1}, \ldots, \bar{g}_{n}$ in the new coordinates, that is

$$
\bar{G}_{i}(z)=\frac{\partial \varphi^{-1}}{\partial x}(\varphi(z)) \bar{g}_{i}(\varphi(z)), \quad i=1, \ldots, n .
$$

In the same fashion, we have

$$
\nabla \bar{H}_{i}(z)=\left(\nabla \bar{h}_{i}\right)(\varphi(z)) \frac{\partial \varphi}{\partial z}(z), \quad i=1, \ldots, n .
$$

Then, for $i=1, \ldots, n$ and $k=1, \ldots, n$, we obtain

$$
\begin{aligned}
\mathcal{L}_{\bar{G}_{i}} \bar{H}_{k}(z) & =\left(\nabla \bar{h}_{i}\right)(\varphi(z)) \frac{\partial \varphi}{\partial z}(z) \frac{\partial \varphi^{-1}}{\partial x}(\varphi(z)) \bar{g}_{k}(\varphi(z)), \\
& =\left(\nabla \bar{h}_{i}\right)(\varphi(z)) \bar{g}_{k}(\varphi(z)), \\
& =\mathcal{L}_{\bar{g}_{i}} \bar{h}_{k}(x) .
\end{aligned}
$$

In the new coordinates $z,(6.22)$ becomes

$$
D \bar{H}_{i}(0)=0, \quad \forall D \in \bar{B}_{d-j-1},
$$

where

$$
\bar{B}_{d-j-1}=\left\{\mathcal{L}_{G_{1}} \circ \cdots \circ \mathcal{L}_{G_{k}}:\left\{G_{1}, \ldots, G_{k}\right\} \subset\left\{\bar{G}_{1}, \ldots, \bar{G}_{n}\right\} \text { and } \sum_{l=1}^{k} w\left(G_{k}\right) \leq j\right\} .
$$

We are now going to prove (6.21) by induction on the order $\|\alpha\|_{r}$. 
Case $\|\alpha\|_{r}=1$

Since $\|\alpha\|_{r}=1$, we necessarily have $|\alpha|=1$ and $\alpha_{i}=0$ if $i=1, \ldots, n_{d-1}$. Hence we have to prove that

$$
\frac{\partial}{\partial z_{k}} \bar{H}_{i}(0)=0, \quad i=n_{d-1}+1, \ldots, n_{d} .
$$

According to Proposition 2.1 in [13], $\bar{G}_{k}(0)=\partial / \partial z_{k}$, for $k=1, \ldots, n$, then by (6.28), we get

$$
0=\mathcal{L}_{\bar{G}_{k}} \bar{H}_{i}(0)=\frac{\partial}{\partial z_{k}} \bar{H}_{i}(0), \quad k=n_{d-1}+1, \ldots, n_{d}
$$

which prove this case.

Case $\|\alpha\|_{r}=k+1$

We assume now that (6.21) holds true for $\alpha \in \mathbb{N}^{n}$, such that $\|\alpha\|_{r} \leq k, k \leq d-j-2$.

Let $D=\mathcal{L}_{G_{1}} \ldots \mathcal{L}_{G_{s}}$ belong to $\bar{B}_{k+1}$. We write

$$
G_{l}(z)=\sum_{k=1}^{n} a_{k}^{l}(z) \frac{\partial}{\partial z_{k}}, \quad l=1, \ldots, s .
$$

The expression

$$
D \bar{H}_{i}(0)=\mathcal{L}_{G_{1}} \ldots \mathcal{L}_{G_{s}} H_{i}(0)
$$

is a sum of terms of the form

$$
a_{i_{1}}^{1}(0)\left(\frac{\partial^{\alpha^{1}}}{\partial z^{\alpha^{1}}} a_{i_{2}}^{2}\right)(0)\left(\frac{\partial^{\alpha^{2}}}{\partial z^{\alpha^{2}}} a_{i_{3}}^{3}\right)(0) \ldots\left(\frac{\partial^{\alpha^{s-1}}}{\partial z^{\alpha^{s-1}}} a_{i_{s}}^{s}\right)(0)\left(\frac{\partial^{\alpha^{s}}}{\partial z^{\alpha^{s}}} \bar{H}_{i}\right)(0)
$$

where, for $l=1, \ldots, s, i_{l} \in\{1, \ldots, n\}$. Furthermore, we have the following property

$$
\sum_{l=1}^{s} r_{i_{l}}=\sum_{l=1}^{s}\left\|\alpha^{l}\right\|_{r}
$$

First, we show that if $\left\|\alpha^{s}\right\|_{r} \geq k+2$ then (6.34) is equal to zero.

According to Theorem 2.1 in [13], a vector field $G \in V$, verify $o(G) \geq-w(G)$ with respect to the weights $\left(r_{i}\right)_{1 \leq i \leq n}$ given by (3.19). If we write $G$ in coordinates, $G(z)=\sum_{k=1}^{n} a_{k}(z) \partial / \partial z_{k}$, then $o\left(a_{i}\right) \geq r_{i}-w(G)$.

Hence if $\left\|\alpha^{l}\right\|_{r}<o\left(a_{i_{l+1}}^{l+1}\right)=r_{i_{l+1}}-w\left(G_{l+1}\right)$, for any $l=1, \ldots, s-1$ or if $r_{i_{1}} \leq w\left(G_{1}\right)$ then (6.34) is equal to zero.

We assume now that

$$
\begin{aligned}
\left\|\alpha^{l}\right\|_{r} & \geq r_{i_{l+1}}-w\left(G_{l+1}\right), \quad l=1, \ldots, s-1, \\
r_{i_{1}} & \leq w\left(G_{1}\right) .
\end{aligned}
$$

We consider the inequality obtained by summing the inequalities $(6.36)$ for $l=1, \ldots, s-1$, we obtain

$$
\sum_{l=1}^{s-1}\left\|\alpha^{l}\right\|_{r} \geq \sum_{l=2}^{s} r_{i_{l}}-\sum_{l=2}^{s} w\left(G_{l}\right)
$$

Adding inequality (6.37) and reordering, we get

$$
\sum_{l=1}^{s-1}\left\|\alpha^{l}\right\|_{r}+\sum_{l=1}^{s} w\left(G_{l}\right) \geq \sum_{l=1}^{s} r_{i_{l}}
$$


Using (6.35), we obtain

$$
\sum_{l=1}^{s-1}\left\|\alpha^{l}\right\|_{r}+\sum_{l=1}^{s} w\left(G_{l}\right) \geq \sum_{l=1}^{s}\left\|\alpha^{l}\right\|_{r}
$$

then

$$
k+1=\sum_{l=1}^{s} w\left(G_{l}\right) \geq\left\|\alpha^{s}\right\|_{r} \geq k+2 .
$$

Thus we must have $\left\|\alpha^{s}\right\| \leq k+1$.

Second, if $\left\|\alpha^{s}\right\|_{r} \leq k$, by the induction hypothesis, we have that (6.34) is equal to zero.

The last case left is $\left\|\alpha^{s}\right\|_{r}=k+1$.

Until now, we have proved that $D \bar{H}_{i}(0)$ is the sum of terms of the form (6.34) with $\left\|\alpha^{s}\right\|_{r}=k+1$ for $D \in \bar{B}_{k+1}$.

Let us consider $E=\left\{\alpha \in \mathbb{N}^{n} \mid\|\alpha\|_{r}=k+1\right\}$ and $E_{l}=\{\alpha \in E|| \alpha \mid=l\}$, then $E$ is the disjoint union of $E_{l}$, $l=1, \ldots, k+1$.

We are going to prove that $\partial^{\alpha} / \partial z^{\alpha} \bar{H}_{i}(0)=0, \alpha \in E_{l}$ for $l=1, \ldots, k+1$, by induction on $l$.

Case $|\alpha|=1$

It is equivalent to prove that $\partial / \partial z_{l} \bar{H}_{i}(0)=0$ for $l=n_{d-j}+1 \leq l \leq n_{d-j+1}$. According to Proposition 3.7, we have $\bar{G}_{l}(0)=\partial / \partial z_{l}, l=1, \ldots, n$, then

$$
0=\mathcal{L}_{\bar{G}_{l}} \bar{H}_{i}(0)=\frac{\partial}{\partial z_{l}} \bar{H}_{i}(0)
$$

Case $|\alpha|=m+1$

We assume that $\partial^{\alpha} / \partial z^{\alpha} \bar{H}_{i}(0)=0$ for $\alpha \in E_{l}, l=1, \ldots, m$, with $m \leq k$. Let $\alpha \in E_{m+1}$, then $\mathcal{L}_{\bar{G}_{1}}^{\alpha_{1}} \ldots \mathcal{L}_{\bar{G}_{n}}^{\alpha_{n}}$ is in $\bar{B}_{k+1}$. Furthermore $\mathcal{L}_{\bar{G}_{1}}^{\alpha_{1}} \ldots \mathcal{L}_{\bar{G}_{n}}^{\alpha_{n}} \bar{H}_{i}(0)$ can be written as the sum of elements in the form (6.34) with $\left|\alpha^{s}\right| \leq m$ plus $\sum_{1 \leq i_{1}, i_{2}, \ldots, i_{s} \leq n} a_{i_{1}}(0) \ldots a_{i_{s}}(0) \partial^{\alpha^{s}} / \partial z^{\alpha^{s}} \bar{H}_{i}(0)$ where $\left|\alpha_{s}\right|=m+1$. By the induction hypothesis the terms with $\left|\alpha^{s}\right| \leq m$ vanish. And since $\bar{G}_{l}(0)=\partial / \partial z_{l}, l=1, \ldots, n$, the only term left is $\partial^{\alpha} / \partial z^{\alpha} \bar{H}_{i}(0)$ which is equal to zero by $(6.28)$.

\subsection{Proof of Theorem 3.12}

Before proving the Theorem, we need the following lemma.

Lemma 6.2. Let $H_{l}$ be a homogeneous function of degree $l$ with respect to the weights (3.19), denote $d H_{l}(0)=$ $\sum_{i=1}^{n} a_{i} d z_{i}$, then $a_{i}=0$ for $i \geq n_{d-l}\left(\right.$ for $l<0$, we set $n_{l}$ as 0$)$.

Proof of Lemma 6.2. First notice that $a_{i} \neq 0$ if and only if $H_{l}$ contains a linear term in $z_{i}$. For $i=1, \ldots, n$, the function $z \rightarrow z_{i}$ is homogeneous of degree $r_{i}=d-n_{i}$, since the weights are decreasing, a homogeneous function of degree $l$ cannot contains linear terms $z_{i}$ for $i \geq n_{d-l}$. Then $a_{i}=0$ for $i \geq n_{d-l}$.

Proof of Theorem 3.12. Let $H: \mathbb{R}^{n} \rightarrow \mathbb{R}$ be the unique function such that $\mathrm{d} H=\omega$ and $H(0)=0$. Since $L_{1}$ is spanned by $\bar{H}_{1}, \ldots, \bar{H}_{n_{1}}$, it is sufficient to prove the Theorem for $H=\bar{H}_{i}, i=1, \ldots, n_{1}$. We prove only the cases $k=0$ and 1 , other cases can be proved in the exact same fashion.

According to Theorem 3.8, $H$ is of order greater or equal to $d-1$ with respect to the weights (3.19), hence we can write

$$
\mathrm{d} H(z)=\mathrm{d} H_{d-1}(z)+\sum_{l_{1} \geq d} \mathrm{~d} H_{l_{1}}(z)
$$


where $H_{l}$ is homogeneous of degree $l$ with respect to the weights (3.19). Similarly, $F$ admit an homogeneous expansion of the form

$$
F(z)=F_{-1}(z)+\sum_{l_{2} \geq 0} F_{l_{2}}(z)
$$

where $F_{l}$ is homogeneous of degree $l$ with respect to the weights (3.19).

Case $k=0$

We apply Lemma 6.2 to the right hand-side of equation (6.43), it comes $\left(\sum_{l_{1} \geq 0} \mathrm{~d} H_{l_{1}}\right)(0)=0$ and $\mathrm{d} \tilde{H}(0)=\mathrm{d} H_{d-1}(0)=\sum_{i=1}^{n_{1}} a_{i} \mathrm{~d} z_{i}$. Evaluating both sides of equation (6.43) at the origin gives the conclusion.

Case $k=1$

Using equations (6.43) and (6.44), we obtain

$$
\begin{aligned}
\mathcal{L}_{F} \omega & =\mathrm{d}\left(\mathcal{L}_{F} H\right), \\
& =\mathrm{d}\left(\mathcal{L}_{F_{-1}} H_{d-1}+\sum_{l_{2} \geq 0} \mathcal{L}_{F_{l_{2}}} H_{d-1}\right)+\mathrm{d}\left(\sum_{l_{1} \geq d} \mathcal{L}_{F_{-1}} H_{l_{1}}+\sum_{l_{1} \geq d, l_{2} \geq 0} \mathcal{L}_{F_{l_{2}}} H_{l_{1}}\right), \\
& \triangleq \mathrm{d}\left(\mathcal{L}_{\tilde{F}} \tilde{H}\right)+\mathrm{d} H_{R} .
\end{aligned}
$$

According to Lemma 2.6, $\left(\mathcal{L}_{\tilde{F}} \tilde{H}\right)$ is of degree $(d-2)$ and $H_{R}$ is the sum of homogeneous functions of degree greater or equal to $(d-1)$. Applying Lemma 6.2 and evaluating both sides of equation (6.47) at the origin, again, gives the result.

\subsection{Proof of Propositions 4.1 and 4.3}

We are going to prove Propositions 4.1 and 4.3 in the $\xi$ coordinates, since the Lyapunov stability is a property independent of the coordinates. For this purpose, we introduce first some notations. We denote system (4.1)

$$
\left\{\begin{array}{l}
\dot{\xi}=\mathfrak{F}(\xi), \\
y=\mathfrak{H}(\xi),
\end{array}\right.
$$

and the error system

$$
\dot{e}=\mathfrak{G}(\xi+e, \mathfrak{H}(\xi))-\mathfrak{F}(\xi),
$$

where $e=\hat{\xi}-\xi$ and $\mathfrak{G}$ is the vector field associated to system (4.9) in the $\xi$ coordinates defined by equation (4.4). We need to state two lemmas before proving Propositions 4.1 and 4.3.

Lemma 6.3. Let $\Phi: \mathbb{R}^{n} \rightarrow \mathbb{R}^{n}$ be a diffeomorphism, homogeneous of degree $d$ with respect to the weights $\left(r_{1}, \ldots, r_{n}\right)$ (as a vector field). Then, the inverse function $\Phi^{-1}$ is homogeneous of degree $-d$ with respect to the weights $r_{i}+d$.

Proof. We have the following identity

$$
\Phi^{-1}(\Phi(x))=x, \quad \forall x \in \mathbb{R}^{n}
$$

Hence

$$
\Phi^{-1}\left(\Phi\left(\lambda^{r_{1}} x_{1}, \ldots, \lambda^{r_{n}} x_{n}\right)\right)=\left(\lambda^{r_{1}} x_{1}, \ldots, \lambda^{r_{n}} x_{n}\right) .
$$

Since the function $\Phi$ is homogeneous, we get

$$
\Phi^{-1}\left(\lambda^{r_{1}+d} \Phi_{1}(x), \ldots, \lambda^{r_{n}+d} \Phi_{n}(x)\right)=\left(\lambda^{r_{1}} x_{1}, \ldots, \lambda^{r_{n}} x_{n}\right) .
$$


Let $\bar{x}=\Phi(x)$, then $\left.x=\Phi^{-1}(\bar{x})\right)$ and

$$
\Phi^{-1}\left(\lambda^{r_{1}+d} \bar{x}_{1}, \ldots, \lambda^{r_{n}+d} \bar{x}_{n}\right)=\left(\lambda^{\left(r_{1}+d\right)-d}\left(\Phi^{-1}\right)_{1}(\bar{x}), \ldots, \lambda^{\left(r_{n}+d\right)-d}\left(\Phi^{-1}\right)_{n}(\bar{x})\right) .
$$

This equality is true for every $\bar{x}$ in $\mathbb{R}^{n}$, since $\Phi$ is a diffeomorphism.

Lemma 6.4. The function $(e, \xi) \mapsto \mathfrak{G}(e+\xi, \mathfrak{H}(\xi))-\mathfrak{F}(\xi)$ can be written as

$$
\mathfrak{G}(e+\xi, \mathfrak{H}(\xi))-\mathfrak{F}(\xi)=E e+\alpha(e, \xi) e+\gamma(e), \quad x, \xi \in \mathbb{R}^{n},
$$

where $E=A-K C, \alpha(e, \xi)$ is a matrix of dimension $n \times n$ such that $\alpha(0,0)=0$ and $\gamma$ is a function of the form

$$
\gamma(e)=\left(\begin{array}{c}
\gamma_{1}\left(\underline{e}_{1}\right) \\
\vdots \\
\gamma_{n}\left(\underline{e}_{n}\right)
\end{array}\right)
$$

with $\underline{e}_{i}=\left(e_{1}, \ldots, e_{i}\right)$ and each $\gamma_{i}: \mathbb{R}^{i} \rightarrow \mathbb{R}$ is linear.

Proof. Denote $R_{F}(z)=F(z)-\tilde{F}(z)$ and $R_{H}(z)=F(z)-\tilde{F}(z)$. By construction, system (6.49) can be written as

$$
\begin{aligned}
\mathfrak{G}(e+\xi, \mathfrak{H}(\xi))-\mathfrak{F}(\xi)= & \text { E.e } \\
& +\frac{\partial \Phi_{h}}{\partial z}\left(\Phi_{h}^{-1}(e+\xi)\right) R_{f}\left(\Phi_{h}^{-1}(e+\xi)\right)-\frac{\partial \Phi_{h}}{\partial z}\left(\Phi_{h}^{-1}(\xi)\right) R_{f}\left(\Phi_{h}^{-1}(\xi)\right) \\
& +\left(R_{h}\left(\Phi_{h}^{-1}(e+\xi)\right)-R_{h}\left(\Phi_{h}^{-1}(\xi)\right)\right) K .
\end{aligned}
$$

But, since $\mathfrak{G}(\xi, \mathfrak{H}(\xi))-\mathfrak{F}(\xi)=0$, we also have

$$
\mathfrak{G}(e+\xi, \mathfrak{H}(\xi))-\mathfrak{F}(\xi)=M e+\alpha(e, \xi) e,
$$

where $M$ is a $n \times n$ real matrix and $\alpha(e, \xi)$ is a matrix of dimension $n \times n$ such that $\alpha(0,0)=0$. Then, all is left to prove is that the linear parts of (6.57) and (6.58) are upper triangular.

We first consider (6.57), it is sufficient to prove that

$$
\frac{\partial\left(\frac{\partial \Phi_{h}}{\partial z}\left(\Phi_{h}^{-1}(e)\right) R_{f}\left(\Phi_{h}^{-1}(e)\right)\right)_{i}}{\partial e_{j}}(0)=0, \quad j \geq i+1 .
$$

By construction, the vector field $z \mapsto R_{f}(z)$ is a sum of homogeneous vector fields of degree higher or equal to 0 , with respect to the weights $r_{i}=n-i, i=1, \ldots, n$. The function $\Phi_{l}$ is homogeneous of degree 0 with respect to the weights $r_{i}=n-i, i=1, \ldots, n$ (as a vector field), thus, according to Lemma 6.3 , the inverse function $\Phi_{l}^{-1}$ is homogeneous of degree 0 with respect to the weights $r_{i}=n-i, i=1, \ldots, n$. We obtain that the vector field

$$
e \rightarrow \frac{\partial \Phi_{h}}{\partial z}\left(\Phi_{h}^{-1}(e)\right) R_{f}\left(\Phi_{h}^{-1}(e)\right)
$$

is a sum of homogeneous vector fields of degree higher or equal than 0 , which gives the result.

We now consider (6.58). It can be proved in the same way than for (6.57). Indeed, by construction the real valued function $z \mapsto R_{h}(z)$ is a sum of homogeneous functions of degree higher or equal to $r_{1}+1=n$ with respect to the same weights $\left(r_{i}\right)_{1 \leq i \leq n}$. Then applying Lemma 6.3 gives the result.

We prove propositions 4.1 and 4.3 in the meantime. Indeed, the first part of the proof is the same for both propositions, only the end is different. 
Proof of Propositions 4.1 and 4.3. We have to prove that the error system

$$
\dot{e}=\mathfrak{G}(e+\xi(t), \mathfrak{H}(\xi(t)))-\mathfrak{F}(\xi(t)), \quad \forall \xi, e \in \mathbb{R}^{n},
$$

is locally asymptotically stable. For this, we consider the Lyapunov function

$$
V(e)=\mathrm{e}^{T} S_{\infty}(\theta) e,
$$

where $S_{\infty}(\theta)$ is the solution of the Riccati equation

$$
\left\{\begin{array}{l}
-\theta S_{\infty}(\theta)-A^{T} S_{\infty}(\theta)-S_{\infty}(\theta) A+C^{T} C=0 \\
S_{\infty}(\theta)^{T}=S_{\infty}(\theta) .
\end{array}\right.
$$

This Lyapunov function verifies:

- $V(0)=0$;

- $V(e)>0, e \neq 0$

- $V$ is of class $C^{1}$.

Now, we need to show that the derivative of $V$ is negative definite along the solutions of system (6.62). According to Lemma 6.4, we can write

$$
\mathfrak{G}(e+\xi(t), \mathfrak{H}(\xi(t)))-\mathfrak{F}(\xi(t))=E e+\alpha(\xi(t), e) e+\gamma(e),
$$

with:

- $E=A-K C$,

- $\alpha(\xi(t), e)$ is a matrix of dimension $n \times n$ such that $\alpha(0,0)=0$,

- $\gamma(e)$ is linear and in a triangular form.

Hence, it gives

$$
\dot{V}_{\mid(6.62)}=-\theta \mathrm{e}^{T} S_{\infty}(\theta) e-(C e)^{2}+2 \mathrm{e}^{T} S_{\infty}(\theta)(\alpha(\xi(t), e) e) 2 \mathrm{e}^{T} S_{\infty}(\theta)(\gamma(e)) .
$$

We overvalue the last two terms in the previous equation. The first over-valuation is done following the same computations as in [9]. The second one is obtained by applying first the Cauchy-Schwarz inequality and then the mean value Theorem. For all $e$ in $\overline{\mathcal{V}}_{2}$, where $\overline{\mathcal{V}}_{2}$ is a neighborhood of the origin that will be set later, we have

$$
\begin{aligned}
\left|\mathrm{e}^{T} S_{\infty}(\theta)(\gamma(e))\right| & \leq n l_{1} C_{1} \sqrt{S}\|e\|_{S_{\infty}(\theta)}^{2} \\
\left|\mathrm{e}^{T} S_{\infty}(\theta)(\alpha(\xi(t), e) e)\right| & \leq\|\alpha(\xi(t), e) e\|_{S_{\infty}(\theta)}\|e\|_{S_{\infty}(\theta)} \\
& \leq\left(\sup _{\substack{\xi \in\{\xi(t), t \geq 0\} \\
e \in \overline{\mathcal{V}}_{2}}}\|\eta(\xi, e)\|_{S_{\infty}(\theta)}\right)\|e\|_{S_{\infty}(\theta)}^{2},
\end{aligned}
$$

with

$$
\begin{gathered}
S=\max _{1 \leq i, j \leq n}\left|S_{\infty}(1)_{i, j}\right| \\
C_{1} \text { is such that }\|x\|_{1, n} \leq C_{1}\|x\|_{S_{\infty}(1)}, \quad \forall x \in \mathbb{R}^{n} \\
\left\|\gamma\left(e^{\prime}\right)-\gamma\left(e^{\prime \prime}\right)\right\| \leq l_{1}\left\|e^{\prime}-e^{\prime \prime}\right\|
\end{gathered}
$$

and $\eta(\xi, e)$ is the derivative of the function $e \mapsto \alpha(\xi, e) e$.

We obtain

$$
\dot{V}_{\mid(6.62)}(e) \leq\left(-\theta+M+\sup _{\substack{\xi \in\{\xi(t), t \geq 0\} \\ e \in \mathcal{V}_{2}}}\|\eta(\xi, e)\|_{S_{\infty}(\theta)}\right)\|e\|_{S_{\infty}(\theta)}^{2}, \quad \forall e \in \overline{\mathcal{V}}_{2} .
$$


Let

$$
\theta^{*}=2+M,
$$

where $M=n l_{1} C_{1} \sqrt{S}$ and take $\theta>\theta^{*}$.

We have to be sure that

$$
\sup _{\substack{\xi \in\{\xi(t), t \geq 0\} \\ e \in \mathcal{\mathcal { V }}_{2}}}\|\eta(\xi, e)\|_{S_{\infty}(\theta)} \leq 1+\left(\theta-\theta^{*}\right) .
$$

Since $\alpha(0,0)=0$, then $\eta(0,0)=0$. In addition, the function $\eta$ is continuous, because all the functions considered here are analytics. Hence, it ensures the existence of two neighborhoods of the origin $\overline{\mathcal{V}}_{1}, \overline{\mathcal{V}}_{2}$ such that

$$
\sup _{\substack{\xi \in \overline{\mathcal{V}}_{1} \\ e \in \mathcal{V}_{2}}}\|\eta(\xi, e)\|_{S_{\infty}(\theta) \leq 1+\left(\theta-\theta^{*}\right) .}
$$

We have proved until now that

$$
\dot{V}_{\mid(6.62)}(e) \leq-\|e\|_{S_{\infty}(\theta)}^{2},
$$

if $\xi(t) \in \overline{\mathcal{V}}_{1}$ for all $t \geq 0$ and $e \in \overline{\mathcal{V}}_{2}$.

Proof of Proposition 4.1. Denote $\overline{\mathcal{V}}_{3}$ a neighborhood of the origin included in $\overline{\mathcal{V}}_{1}$ such that for all $x, y \in \overline{\mathcal{V}}_{3}$, $(x-y) \in \overline{\mathcal{V}}_{2}$. Then $\lim _{t \rightarrow+\infty}[\xi(t)-\hat{\xi}(t)]=0$ if $\xi(0), \hat{\xi}(0) \in \overline{\mathcal{V}}_{3}$ and if the set $\overline{\mathcal{V}}_{1}$ is positively invariant under system (6.48).

Finally, we define $\mathcal{V}_{1}=\varphi \circ \Phi_{h}^{-1}\left(\overline{\mathcal{V}}_{1}\right)$ and $\mathcal{V}_{2}=\varphi \circ \Phi_{h}^{-1}\left(\overline{\mathcal{V}}_{3}\right)$. The sets $\mathcal{V}_{1}$ and $\mathcal{V}_{2}$ are neighborhoods of the origin and $\overline{\mathcal{V}}_{1}$ is positively invariant under system $(6.48)$ if and only if $\mathcal{V}_{1}$ is positively invariant under system (4.1) since the map $\xi \mapsto \varphi \circ \Phi_{h}^{-1}(\xi)$ is a diffeomorphism.

Proof of Proposition 4.3. The origin of system (6.48) is Lyapunov stable since Lyapunov stability is invariant under change of coordinates, thus there exists a neighborhood of the origin $\overline{\mathcal{V}}_{4}$ such that any solution of system (6.48) starting in $\overline{\mathcal{V}}_{4}$ stays in $\overline{\mathcal{V}}_{1}$. Let $\overline{\mathcal{V}}_{3}$ be a neighborhood of the origin included in $\overline{\mathcal{V}}_{4}$ such that for all $x, y \in \overline{\mathcal{V}}_{3},(x-y) \in \overline{\mathcal{V}}_{2}$. We then obtain that $\lim _{t \rightarrow+\infty}[\xi(t)-\hat{\xi}(t)]=0$ if $\xi(0), \hat{\xi}(0) \in \overline{\mathcal{V}}_{3}$. Finally, we define $\mathcal{U}=\varphi \circ \Phi_{h}^{-1}\left(\overline{\mathcal{V}}_{3}\right)$ which is a neighborhood of the origin since the map $\xi \mapsto \varphi \circ \Phi_{h}^{-1}(\xi)$ is a diffeomorphism.

\section{Conclusion}

In this paper, we have constructed a homogeneous approximation for systems with multi-output and without input, satisfying an observability rank condition. This approximation retains pertinent information relative to observability, particularly, it has the same observability space as the original system. The construction given here is not always easy to compute, even if it is explicit. But, we have exhibited sufficient conditions which give some degree of freedom for the construction.

This approximation has been further used for the design of a local observer which has been shown to be convergent for Lyapunov stable systems. Once the change of coordinates is obtained, the observer design is straight and simple.

The performance of the proposed method has been illustrated on an example and compared with linear approximation. It appears that our observer presents a substantially wider domain of convergence for this system.

Acknowledgements. The authors are deeply grateful to Claude Moog from IRCCyN, Gildas Besançon from GIPSA-Lab, Witold Respondek from LMI and Alessandro Astolfi from EEE Dept, Imperial College of London, for their help and advices on the work presented here. 


\section{REFERENCES}

[1] V. Andrieu and L. Praly, On the existence of a Kazantzis-Kravaris/Luenberger observer. SIAM J. Control Optim. 45 (2006) 432-456.

[2] V. Andrieu, L. Praly and A. Astolfi, Homogeneous approximation, recursive observer design, and output feedback. SIAM J. Control Optim. 47 (2008) 1814-1850.

[3] G. Besançon and A. Ticlea, An immersion-based observer design for rank-observable nonlinear systems. IEEE Trans. Autom. Control 52 (2007) 83-88.

[4] R.M. Bianchini and G. Stefani, Graded approximations and controllability along a trajectory. SIAM J. Control Optim. 28 (1990) 903-924.

[5] G. Conte, C.H. Moog and A.M. Perdon, Algrebraic Methods for Nonlinear Control Systems. Springer, London, 2nd (2007).

[6] S. Diop and M. Fliess, Nonlinear observability, identifiability and persistent trajectory, in IEEE 30th Conference on Decision and Control (1991) 714-719.

[7] M. Fliess, C. Join and H. Sira-Ramirez, Nonlinear estimation is easy. Int. J. Model. Identification Control. 4 (2008) 12-27.

[8] J.P. Gauthier and G. Bornard, Observability for any u(t) of a class of nonlinear systems. IEEE Trans. Autom. Control 26 (1981) 922-926.

[9] J.P. Gauthier, H. Hammouri and S. Othman, A simple observer for nonlinear systems applications to bioreactors. IEEE Trans. Autom. Control 37 (1992) 875-880.

[10] A. Glumineau, C.H. Moog and F. Plestan, New algebraic-geometric conditions for the linearization by input-output injection. IEEE Trans. Autom. Control 41 (1996) 598-603.

[11] R.W Goodman, Nilpotent Lie groups: structure and applications to analysis, Lectures Note Math. Springer-Verlag, Berlin, New-York 562 (1976).

[12] H. Hermes, Control systems which generate decomposable lie algebras. J. Differ. Equ. 44 (1982) 166-187.

[13] H. Hermes, Nilpotent and high-order approximations of vector-field systems. SIAM Rev. 33 (1991) $238-264$.

[14] M. Kawski, Homogeneous stabilizing feedback laws. Control Theory and Advanced Technology 6 (1990) 497-516.

[15] N. Kazantzis and C. Kravaris, Nonlinear observer design using Lyapunov's auxiliary theorem. Syst. Control Lett. 34 (1998) 241-247.

[16] H.K. Khalil, High-gain observers in nonlinear feedback control. In International Conference on Control, Automation and system (2008).

[17] A.J. Krener and A. Isidori, Linearization by output injection and nonlinear observers. Syst. Control Lett. 3 (1983) 47-52.

[18] A.J. Krener and W. Respondek, Nonlinear observers with linearizable error dynamics. SIAM J. Control Optim. 23 (1985) 197-216.

[19] J. Li and C. Qian, Global finite-time stabilization by dynamic output feedback for a class of continuous nonlinear systems. IEEE Trans. Autom. Control 51 (2006) 879-884.

[20] T. Menard, E. Moulay and W. Perruquetti, A global high-gain finite-time observer. IEEE Trans. Autom. Control 55 (2010) 1500-1506.

[21] K. Nam, An approximate nonlinear observer with polynomial coordinate transformation maps. IEEE Trans. Autom. Control 42 (1997) 522-527.

[22] A.R. Phelps, On constructing nonlinear observers. SIAM J. Control Optim. 29 (1991) 516-534.

[23] L. Rothschild and E. Stein, Hypoelliptic differential operators and nilpotent groups. Acta Mathematica 137 (1976) $247-320$.

[24] I. Souleiman, A. Glumineau and G. Schreier, Direct transformation of nonlinear systems into state affine miso form for observer design. IEEE Trans. Autom. Control 48 (2003) 2191-2196.

[25] G. Stefani, Polynomial approximations to control systems and local controllability, in Proc. of the 24th IEEE Conference on Decision and Control (1985) 33-38.

[26] V. Sundarapandian, Local observer design for nonlinear systems. Math. Comput. Model. 35 (2002) 25-36.

[27] H.J. Sussmann, A general theorem on local controllability. SIAM J. Control Optim. 25 (1987) 158-194. 